
\title{
Besearch S Surare \\ An Empirical Analysis of Software Fault Proneness Using Factor Analysis with Regression
}

Deepak Sharma ( $\nabla$ deepak.usict.9000501@ipu.ac.in )

Guru Gobind Singh Indraprastha University

\section{Pravin Chandra}

Guru Gobind Singh Indraprastha University

\section{Research Article}

Keywords: Soft computing, Bugs, Faults, Prediction, Latent variables, Factor with Regression Analysis

Posted Date: September 13th, 2021

DOl: https://doi.org/10.21203/rs.3.rs-832897/v1

License: (9) This work is licensed under a Creative Commons Attribution 4.0 International License. Read Full License 


\title{
An Empirical Analysis of Software Fault Proneness Using Factor Analysis with Regression
}

\author{
Deepak Sharma ${ }^{1}$ Pravin Chandra ${ }^{2}$
}

\begin{abstract}
During the early stages of the life cycle development process for software, the developer mainly makes use of the fault prediction process for the development of different modules. These modules help in detecting faulty modules and classes. Further, this process also helps in determining the modules which require a high level of refactoring during the maintenance stage. The objective of this research is to classify faults and to explore the usability of Factor Analysis with Regression (FAWR) which drastically ameliorate the system performance. A review of recent studies performed that uses the different fault prediction techniques. To direct this research, two research questions (RQ) are defined, one related to the integration of techniques to enhance the development of fault prediction model, and another is to check the technique to overcome the limitations of old methods. To answer these RQs, FAWR techniques are used for predicting faults. To assess the quality of the technique, two experiments were conducted. Results show that FAWR is the better performing method among the two prediction methods investigated. The results proved that the prediction capability of FAWR technique is significantly better. Factorization method is able to classify a module whether it is fault-prone or not. The constructed models use to estimate the proneness of faults surpass the standard regression models. The system evaluations indicate that the reduction of terms results in the betterment of outcomes. Moreover, the consideration of FAWR is a significant technique for the prediction of faults in software.
\end{abstract}

Keywords: Soft computing, Bugs, Faults, Prediction, Latent variables, Factor with Regression Analysis

\section{Introduction}

Recent software systems are continuously increasing in complexity and size, assuring their dependability and quality, which is dependent on software flaws, is critical. Fault prediction in software actively assists in problem detection by exposing likely faulty code regions in the software system. This assessment of code sections that are more prone to errors can assist the testing team in allocating software quality assurance resources more effectively. Several studies have looked into SFP modelling because of its inherent benefits in optimizing testing resource use and enhancing software project quality. Various learning approaches have been extensively used for SFP throughout the previous two decades. There are a few of them: Regression approaches, k-nearest neighbours algorithm, Nave Bayes, Neural Network, decision trees, rule-based techniques, and so on. However, an evaluation of these algorithms revealed that the majority of them had an average prediction accuracy of 85 percent to 90 percent, with a higher level of misclassification. Furthermore, performance has been inconsistent across dataset of fault.

When it comes to software systems, the majority of the flaws are focused in a tiny section of code. As a result, employin g accuracy measurements to evaluate a classification method will not provide realistic picture of the model's performanc e. Individual classification and learning approaches have reached the edge of their performance thresholds, according to previous research in the SFP area (rathor and kumar 2017, sharma and Chandra 2018, 2019a), and their performance cannot be enhanced further without external modifications in the fault datasets or model building process. Researchers (rathor and kumar 2017) have endeavored to crack this performance measure by executing different performance measures further developing methodologies, for example, advancing the data content of preparing training datasets, modifying the forecast model to explicit nearby business objectives, and consolidating various datasets of metrics. SFP models. SFP models based on ensemble algorithms have recently acquired traction in the software engineering area. For SFP, only a few scholars (Sharma and Chandra 2019a) have looked at regression approaches including linear regression, MLR, and stepwise regression. These research, however, were confined to a few fault datasets and only looked at one or two regression approaches.

\footnotetext{
$\square^{1}$ deepak.usict.9000501@ipu.ac.in

(corresponding author)

${ }^{2}$ coe@ipu.ac.in

${ }^{1,2}$ University School of Information, Communication, and Technology, Guru Gobind Singh Indraprastha University, Delhi, India
} 
These performance-improving methods yielded promising results in terms of breaking the performance bottleneck in Furthermore, the researchers have reported on a number of new and improved regression approaches, but no assessment of these techniques for the SFP has yet been completed. This prompted us to investigate these regression approaches and determine their applicability to the SFP. This work conducts a comprehensive experimental evaluation of all factor analysis regression approaches, including simple regression, linear regression, MLR, stepwise regression, and MLR with interaction for the SFP. Most of the approaches employed in this work have never been fully examined for the SFP, to our knowledge. Two distinct regression models, MLR with interaction(WI) and MLR (WI)with square terms, were chosen as predictors for the FAWR methods. The experiment is carried out on five publicly accessible software fault datasets from the PROMISE data repository. The performance of FAWR methods is evaluated using $\mathrm{R}$ square and $\mathrm{R}$ square adjusted metrics. The performance of FAWR methods is evaluated using R square and R square adjusted metrics. The $\mathrm{R}$ square and $\mathrm{R}$ square adjusted tests are used to evaluate the relative significant difference in the performance of two distinct FAWR methods. In addition, a comparison study is performed to determine the success of the approaches employed in terms of reducing software testing costs and increasing efficacy. This empirical study's findings and observations can assist practitioners in developing successful SFP models.

\section{Related work}

Our day-to-day life gets highly influenced by various kind of software and the quality of the software further depends on the growing demand of the society and their needs (Erturk and Sezer 2015; Turhan et al. 2009). According to some researchers quality of the software is mainly reflected in the level of reliability of the system, higher reliability means a higher quality of the software, and lower probability for the occurrence of failures in the system, and vice versa (Jabangwe et al. 2015; Saxena and Saini 2011; Basili et al. 1996; Briand et al. 2001; Subramanyam and Krishnan 2003; Gyimothy et al. 2005). Fault in any system occurs due to the errors, a high number of errors means higher faults (Al Dallal 2018; Colanzi et al. 2020) and lower reliability (Al Dallal 2012a; Mockus and Weiss 2002), and accordingly low quality of software's are not desired for the need of the peoples(Chandrasekaran et al. 2010; Turhan et al. 2009; Hassan 2009). Hence, to improve the quality of the system, the developer needs to work on reducing the number of failures in the system, or the frequency of occurring faults in the system (Zadeh 1996; Chaturvedi 2008; Mohanty et al. 2010). The major reason for the failures in the software are errors. An error occurs due to lost human action which causes any mistake in the software (Chidamber and Kemerer 1994; Jabangwe et al. 2015). These mistakes are termed as errors if they incurred during the implementation phase of the software (Panichella et al. 2016; Di Nucci et al. 2018). Fault can be defined as the inaccurate coding, actions, processes, wrongly define data or any physical defect in the software or the hardware(Olague et al. 2007; Shatnawi and Li 2008; Aggarwal et al. 2009). Consequently, mistakes lead to errors, errors lead to faults and faults lead to failures (Saxena and Saini 2011; Basili et al. 1996; Briand et al. 2001; Subramanyam and Krishnan 2003). Failure is when we run the software and the "defect" term displayed on the system (Singh et al. 2010). Failure is when we run the software and the "defect" term displayed on the system (Singh et al. 2010). Software fault prediction(SFP) has been labeled as a subject of study, which is purely focused on the prediction of faults in the system, based on historical data and other metrics related to the modules (Al Dallal 2011). Software fault prediction can be a process under which modules are developed which can help in the identification of modules that require further testing or refactoring and this process is a part of the early stage of the development life cycle (Subhashis Chatterjee and Shukla 2016). The software testing gets refined if the developer makes use of software fault prediction, and improves the maintenance phase by tracing the modules which need to be refactored (Dagpinar and Jahnke 2003). It also reduces the time and efforts involved in the review process of codes and ultimately lead to the selection of the accurate software design and development of a stable software system (Al Dallal 2013). The term reliability in the software development process was for the first time introduced in 1970, and later on software developers have started showing interest and researching the area of software fault prediction (Al Dallal and Morasca 2014). Many modern techniques that we use today for software fault prediction are the results of the long-term evolution of software reliability (Kosker et al. 2009). Two major metrics have been used by researchers for the software fault prediction, one is object-oriented and another is static code (Staron et al. 2018). Studies have shown that static code is less impactful in software fault prediction as compared to object-oriented metrics (Yamada et al. 1992). Object-oriented metrics mainly include the structural features of the software system such as; coupling, inheritance, complexity, cohesion, encapsulation, and size of the metrics as well (Goel and Okumoto 1979; Kapur et al. 2011; Schneidewind 1975; Yamada et al. 1983). The inception of research work selected from the year 1993 to 2019 depicts in Fig 1 explains the details of the year wise publications (Chiang et al. 2020; Goel and Okumoto 1979; Huang and Lyu 2005; Kapur et al. 2011; Karnavas et al. 1993; Killick and Eckley 2014; Kitchenham et al. 2001; Liang Yin and Trivedi 1999; Pillai and Sukumaran Nair 1997; Schneidewind 1975; Staron et al. 2018; Wohlin et al. 2012; Xie et al. 2007; Xie and Zhao 1992; Yamada et al. 1983, p., 1992). 
Figure 1 shows that the average number of studies conducted every year was 4. It also indicates that the trend of conducting studies on using modern computing methods for SFP has increased over the years. The highest number of study were published in the 2017. This indicates the increased interest of researchers in exploring computing techniques (Subhashis Chatterjee and Roy 2015; Dejaeger et al. 2013; Goyal et al. 2013; Lo et al. 2005; Ramani et al. 2012)for software fault prediction. The minimum number of research work was published from 1993 to 1999 (S. Chatterjee et al. 2017; Chidamber and Kemerer 1994; Staron et al. 2018).

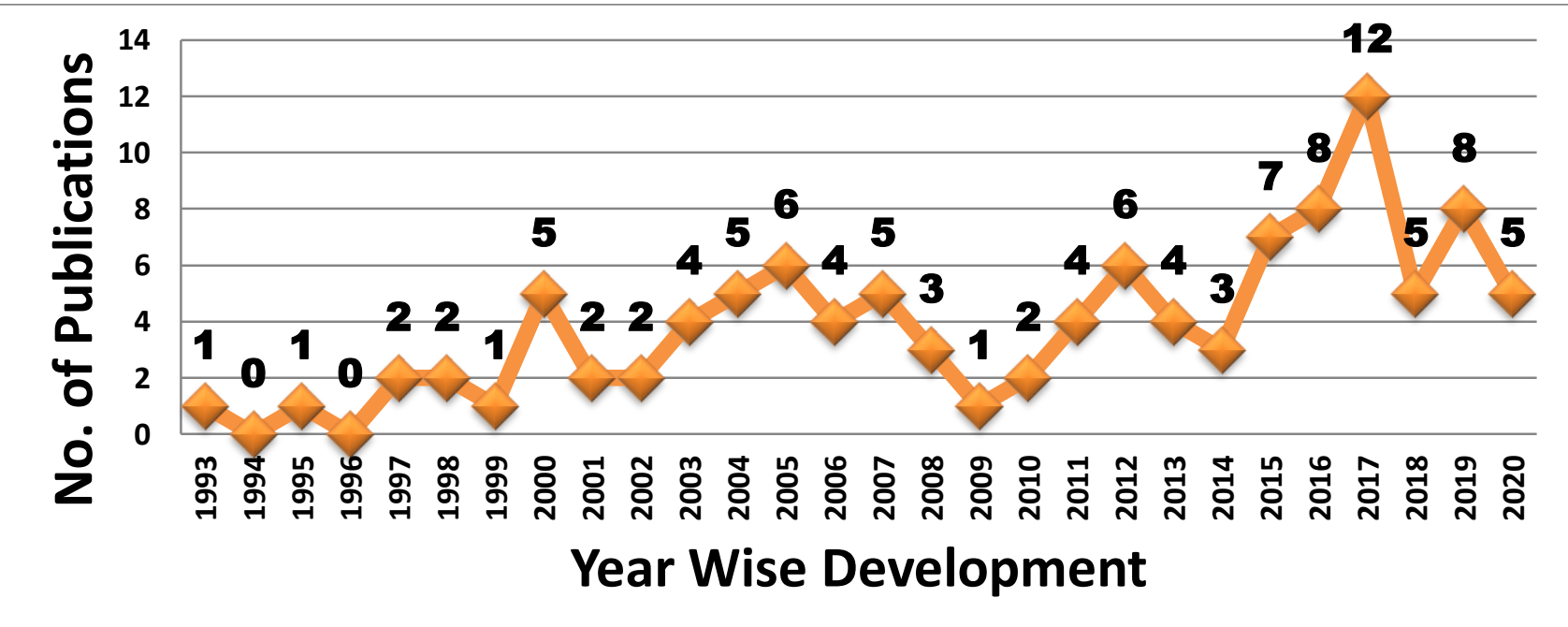

Fig. 1 Taxonomy of year wise development of techniques and their publications

\section{Problem Statement with Research questions}

A large portion of the past issue fault prediction techniques depend on characterizing, programming languages, and module of the software into a defective or non-faulty module, and this kind of prediction doesn't offer enough coordination to adequately disperse the necessary resources. A class of the defective programming modules may have errors in comparison with different modules and may require a couple of more endeavors to be fixed. Hence, indicating the resources completely dependent on the class of modules i.e. faulty or non-faulty. This may also bring the inefficient utilization of resources. Hence, anticipating the prediction of percentage (\%) of errors and their occurrence in programming languages gives the probability that decides shortcomings that will emerge inside the given software project, and this sort of prediction can be extremely valuable to limit the efforts of testing on the modules that have additional errors.

RQ1 How does the integration of techniques enhance the SFP model? Moreover, how does the combination of techniques enhance the existing state of the art in terms of SFP?

RQ2 How does the proposed approach overcome the limitations of previous SFP methods?

\section{Structure of the data}

In this research, the combinations of Chidamber and Kemerer CK(6) i.e. lack of cohesion (LCOM), no. of children (NOC), the coupling between objects (CBO), Depth of Inheritance Tree (DIT), response for the classes (RFC), weighted methods (WMC) and Object-Oriented (OO(11)) class level metrics (Al Dallal 2012a; Chandrasekaran et al. 2010; Zadeh 1996) i.e. OO_FANIN number of other classes that reference the class, OO_NOPRM number of private methods, OO_NOPA number of public attributes, OO_NOPM number of public methods, OO_NOAI number of attributes inherited, OO_NLOC number of lines of code, OO_NOM number of methods, OO_NOMI number of methods inherited, OO_NOPRA number of private attributes, OO_FANOUT number of other classes referenced by the class, OO_NOA number of attributes dataset has been used. The five different software modules i.e. Eclipse(E1), Lucene(L1), Mylyn(M1), Equinox(E2) and PDE(P1) contains in dataset (Chinna Gounder Dhanajayan and Appavu Pillai 2017; Sharma and Chandra 2018a, 2018b, 2019b, 2020a). To construct a robust model the open-source bug prediction measurements (metrics) were analyzed. 
Table 1 explains the details of the dataset i.e. modules, metrics factors, etc. utilized for model development and model verification. All five different software modules have a unique number of defects, classes, modules, and attributes.

Table 1 Summary of the dataset

\begin{tabular}{|c|c|c|c|c|c|c|}
\hline \multicolumn{2}{|c|}{$\mathrm{CK}(6)+\mathrm{OO}(11)=17$ Attributes } & \multirow{2}{*}{$\begin{array}{c}\text { Total } \\
\text { Factors }\end{array}$} & \multirow{2}{*}{$\begin{array}{c}\text { Short Name of } \\
\text { software modules. }\end{array}$} & \multirow[t]{2}{*}{ Total Classes } & \multirow{2}{*}{$\begin{array}{c}\text { Total } \\
\text { Defects }\end{array}$} & \multirow[t]{2}{*}{ Total Modules } \\
\hline $\begin{array}{l}\text { Attributes in } \\
\text { CK[6] }\end{array}$ & Attributes in $\mathrm{OO}[11]$ & & & & & \\
\hline $\mathrm{CBO}(\mathrm{C} 1)$ & FANIN(F1), FANOUT(F2), & 15 & E1 & 997 & 374 & 997 \\
\hline DIT(D1), & NOA(N2), NOAI(N3), & & L1 & 691 & 97 & 691 \\
\hline LCOM(L1), & NLOC(N4), NOM(N5), & & M1 & 2196 & 340 & 1862 \\
\hline $\begin{array}{l}\operatorname{NUC}(\mathrm{N} 1) \text {, } \\
\mathrm{RFC}(\mathrm{R} 1) \text { and }\end{array}$ & $\begin{array}{l}\text { NOMI(N6), NOPRA(N7), } \\
\text { NOPRM(N8), NOPA(N9) }\end{array}$ & & E2 & 439 & 244 & 324 \\
\hline WMC(W1) & and $\mathrm{NOPM}(\mathrm{N} 10)$ & & P1 & 1562 & 341 & 1497 \\
\hline
\end{tabular}

\section{Research Methods}

This section describes an overview of the techniques that are used in this study, which clarifies all the inputs and outputs parameters with procedural phases. The proposed technique is the SFP model consists of combining the Factor Analysis (FA) with regression (Cohen 2013; Lawley and Maxwell 1973; Runkler 2012; Sharma and Chandra 2017, 2019a) and its measurements as well as their separate comparisons. This technique will be used for the first time to predict faults.

Fig.2 Overall design of the software fault prediction technique

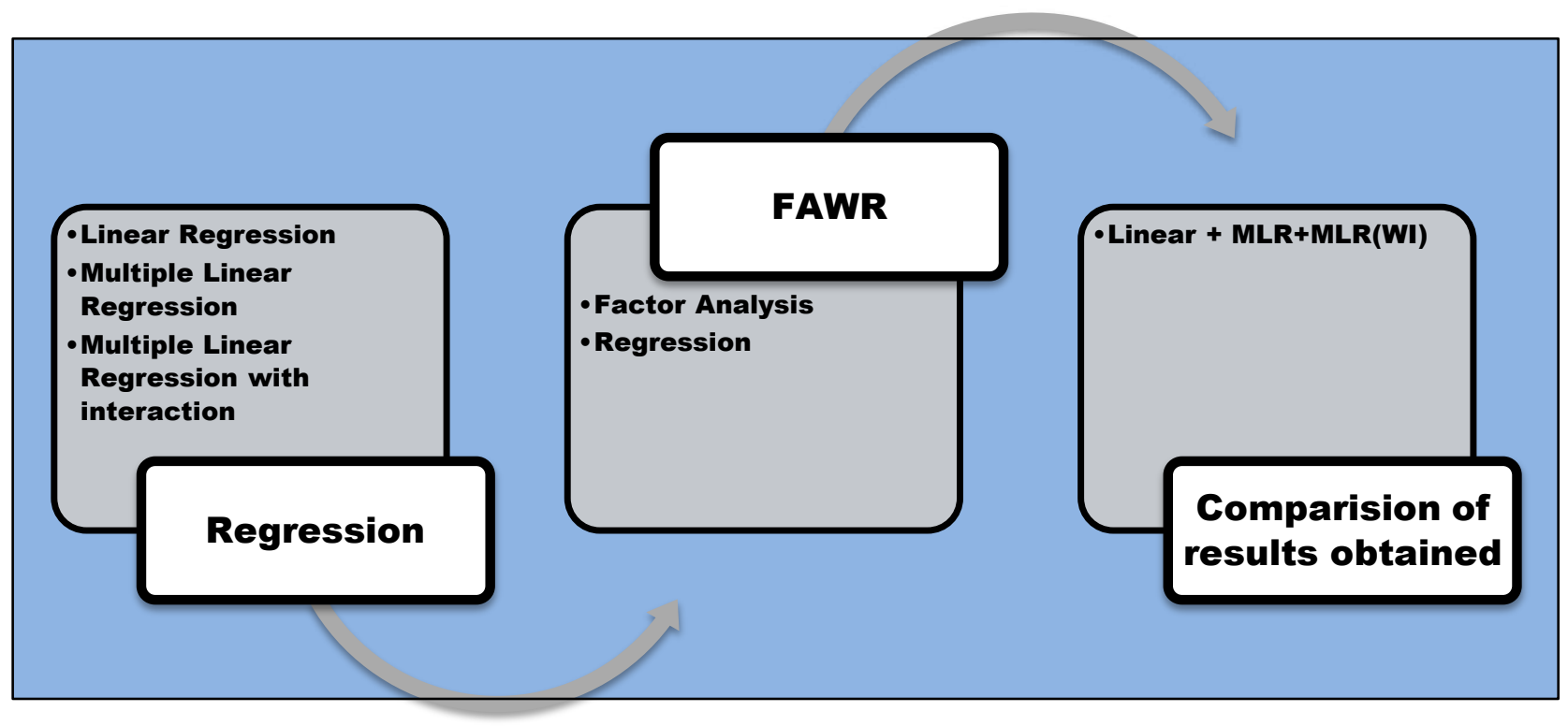

\subsection{Fault prediction based on Regression methods and factor analysis}

In this section, we have introduced the regression methods and described the different interaction analysis based on linear analysis and described the exploratory factor analysis. Then, we proposed a robust technique based on FAWR (Cohen 2013; Lawley and Maxwell 1973; Runkler 2012; Sharma and Chandra 2019a), which is more efficient for the model development of fault predictor of software based on the ingrained property of the data sets. Simple regression used in the current study, mainly established the relationship between the response variable and the predictor variable, using a linear regression line which is a straight line, and the relationship between variables can be visualized through this line. The researcher has used the following regression equation(1) 


$$
\mathbf{Z}=\mathbf{C}+\boldsymbol{\beta} \mathbf{Y}_{+} \mathbf{E}
$$

In this study, we have considered Equation 1 having a single variable, denoted by Z; to predict the fault proneness. $\mathrm{C}$ is the "intercept", which is constant; and the value of the response variable is calculated using this intercept if the independent variable is set to zero or equal to zero. " $\beta$ " is the slope or the regression coefficient and is also called the multiplier. This defines the percentage change in the response variable if there is a unit change in the predictor variable. $\mathrm{E}$ is an error component that is assumed to be independent. It is normally distributed with zero mean value and $\sigma^{2}$ variance. Define $Y$ as well. Linear regression is generally used as it is the most common and easiest way to establish the relationship between two such variables, where one gets affected by another variable, or a change in one can cause a change in another variable. It indicates both the direction and the degree of the cause and effects the relation (Runkler 2012). The method can be used only when both the predictor and response variables have a linear relationship, which means the change in the response variable due to change in the predictor variable by a constant unit. Due to simplicity, it the best-opted method over others for analysis, especially when the scale of the sample is small. The results analyzed by this method are easy to understand and interpret. Though, the regression model can also fail, if we add a huge number of predictor variables in the regression equation as it makes the results more complicated to interpret and analyze. Individual impacts of each predictor variable to be known are somewhat complex in the regression model with a large number of predictor variables. A significant percentage of variation is explained by the regression model, which is caused by the predictor variable, and this percentage of variation helps in determining whether the model is fit or not, whether the dataset needs to enlarge or whether the variables used need to refined and re-selected. Sometimes the relation between variables is underestimated but still, the presence of relation between variables gets proved using this method (Cohen 2013). Hence, to establish a relationship using the regression model is more important than the percentage of variation, and this has been used as the initial and basic step to check for the development of the processes further. The testing of a linear relationship between variables is most important to be checked before any further analysis. Though, linear regression takes under consideration any expected nonlinearity or the known nonlinearity, if the variables entered in the regression equation get transformed before applying regression. In linear regression, there can be one or more than one predictor variable, and still, the model can be developed with ease.

\subsection{Multiple Linear Regression (MLR)}

Multiple regression is a statistical tool used to predict the value of the response variable, using a set of explanatory variables and fitting the standard errors to be minimum. The estimated errors are minimized and the model is considered to be best to fit using the sum of squared error values (Sharma and Chandra 2019). The main problem researchers have faced is related to the selection of the set of explanatory variables, to form a linear relationship. Highly correlated or highly uncorrelated variables in the set of explanatory variables which cause the failure of the linear regression model. The objective or goal of the multiple linear regression is to reduce the number of explanatory variables and minimize the errors in the model, with $n$ number of observations in the dataset. Multiple regression is mostly used in the behavioral sciences, due to its flexible features and power to accommodate the " $\mathrm{n}$ " number of predictor variables. Behavioral scientists make use of this method, for answering a wide variety of questions, and establish the cause and effect relations. Both the response and the predictor variables should be quantitative for applying multiple regression. Personality traits, skills, or the abilities are taken as quantitatively and it can be used as the predictor variables apart from categorical measures such as; gender, age group and income (Cohen 2013). The dependent variable is mostly used in the study as a continuous variable while the power of multiple regression is both categorical and quantitative for regression equation formulation (Coxe et al. 2012) (Aiken et al. 2012). Further, the relationship between variables can be linear, or curvilinear which mainly depends on the interactions between variables (Cohen 2013; Lawley and Maxwell 1973; Runkler 2012; Sharma and Chandra 2017, 2019a). The response variable is also called the criteria, and this is based on the fact that the score of the predictor variables helps in setting a criterion for the linear relationship. The fitness of the multiple regression model (MRM) depends on the ability of the predictor variables to account for the criterion. Further, the unique contribution of each predictor variable can be known from the results, and the comparison can be done among the predictor variables also, related to the contribution of each predictor variable in the criterion. Assessing the unique contribution of a predictor in the set of predictors, and the subsets among each predictor they can further enhance the application and utility of the multiple regression analysis. Multiple linear regression is mainly used to summarize the relationship between the predictors and the criterion for a specific time such as; the impact of job satisfaction 
measured at a certain point of time on the performance of employees for that period only. Further, the value of response or criterion can be predicted based on the predictor variables and the coefficients of the predictor variables (Runkler 2012). The variables are taken based on the previous well-established models, or theories, and similarly, the hypothesis is formulated which can be tested using the regression values. The equation for the multiple predictor variables for determining relation is given below:

$$
\mathbf{Y}=\mathbf{C}+\boldsymbol{\alpha}_{1} \mathbf{x}_{1}+\boldsymbol{\alpha}_{2} \mathbf{x}_{2}+\ldots \ldots \ldots \boldsymbol{\alpha}_{\mathrm{n}} \mathbf{x}_{\mathrm{n}+\mathrm{E}}
$$

In this study, we have considered Equation 2 having a multiple variables, denoted by $\mathrm{Y}$; to predict the fault proneness. C is the "intercept", which is constant; and the value of the response variable is calculated using this intercept if the independent variable is set to zero or equal to zero. " $\boldsymbol{\alpha}$ " is the slope or the coefficients for the independent variables i.e $\mathrm{X} 1, \mathrm{X} 2 \ldots \mathrm{Xn}$ and is also called the multiplier. This defines the percentage change in the response variable if there is a unit change in the predictor variable. $\mathrm{E}$ is an error component that is assumed to be independent. Here, the value of the response variable can be determined as the sum of the intercept value and the value of other predictor variables. If all the predictor variables will be considered as zero, then the value of the intercept will be equal to the value of the response variable. The value of each predictor variable will be a multiplication of their noise or actual value and the coefficient of that particular predictor variable. Simple linear regression is not as powerful as multiple regression because it has the power to explain the variation caused by multiple predictor variables in one response variable. This is also interpreted in the same way as simple linear regression.

\subsection{With Interaction(WI)}

In multiple regression, the interaction between two variables is termed as a function that is tested to accept or reject the hypothesis formulated on the past model, literature, or some theory (Lawley and Maxwell 1973; Runkler 2012). This interaction is determined as a product of the values of predictors, entered for interaction. Say if two predictor variables are taken for the interaction purpose. i.e. $\mathrm{X}$ and $\mathrm{Z}$ variables, then the variable $\mathrm{XZ}$ will reflect the interaction between the variables. The regression equation number 3 for interaction using the same example of interaction will be as follows:

$$
Y=b 0+b 1 X+b 2 Z+b 3 X Z
$$

\subsection{Self-Interaction (SI)}

The interaction will have a partial effect only if both the predictor variables are also taken in the regression equation along with the interaction variable. The main effect is due to the predictor variables except the interaction variables. Hence, only the interaction effect can be known by the coefficient of interaction variables, while the total effect will be calculated as the main effect and the interaction effect. Given equation 4 considered when we calculate the effect of linear terms with self-interaction terms on dependent variable $\mathrm{X}$.

$$
X=\sum_{n=1}^{K} b_{n} \mathbf{z}_{n}+\sum_{i=1}^{K} \sum_{j=i+1}^{K} \alpha_{i j} \mathbf{z}_{i} \mathbf{z}_{j}
$$

where, $b_{n}$ : coefficients for the independent variables $\mathrm{z}_{\mathrm{i}} \mathrm{z}_{\mathrm{j}}$

$\alpha_{\mathrm{ij}}$ : coefficients of interaction between two different independent variables $\mathrm{z}_{\mathrm{i}} \mathrm{z}_{\mathrm{j}}$

K: no. of variables.

\subsection{Self-Interaction (SI) with (Square Terms)}

In this study, we have considered equation number 5 having set of linear terms, multiple variables, interaction of linear terms and square of linear terms, denoted by F; to predict the fault proneness. 


$$
F=\sum_{n=1}^{N} c_{n} z_{n}+\sum_{i=1}^{N} \sum_{j=i+1}^{N} \beta_{i j} z_{i} z_{j}+\sum_{k=k+1}^{N} \alpha_{k} z_{k}^{2}
$$

where, $C_{n}$ : coefficients for the independent variables $Z_{n}$. $\beta_{i j}$ : coefficients of interaction between two different independent variables $Z_{\mathrm{i}} Z_{\mathrm{j}}$. $N$ : no. of variables. $\alpha_{\mathrm{K}}$ : coefficients for the square of independent variables $Z_{k}$.

\subsection{Factor Analysis}

Identification of the relationship between the variables is considered to be an essential part of any scientific or behavioral science research (Lawley and Maxwell 1973; Runkler 2012; Sharma and Chandra 2019). For any method used for the analysis, the variables should be well defined and only those variables which have already been tested and found to be reliable in the literature for establishing the relationship. The variables should be well proved by the scientific study. Further, the relationships of some variables are already proved in terms of theory or models. Hence, we conduct a study on some new area, taking new variables that have no or very few literatures available, need to be defined in a precise manner, as the nature and extent of the relationship between variables might not be proved by scientists. Hence, the clarification on all the variables is important and any pre-assumptions regarding the relationship between variables is not possible. In such a situation, one of the method which is considered to be the best-applied model is factor analysis, as it establishes the relationship between the variables which are newer in the scientific area and needs to be related to each other to study the characteristics of the variables. Factor analysis helps in defining the variables more precisely based on their homogenous characteristics, and variation percentage. Further, the complex relationships also got exposed through factor analysis, and these variables are omitted from the study.

An increasing trend can be seen in the studies related to physical sciences and behavioral sciences for using factor analysis to group the variables based on their homogenous features under different factors, to establish the relationships between variables and development of the model as well. The analysis itself is complex and requires a deep understanding of mathematics. To use factor analysis, the researcher should possess a sound mathematical knowledge as well as proper training to run this analysis (Browne 1969; Harman and Chomsky 1967; Whitely 1981). Another important aspect of factor analysis is that it makes use of both mathematics and empirical science. Hence, the effective use of the factor analysis can be done by the researchers having a clear concept of the mathematical aspects as well as empirical science. In this study, we have considered equation 6 having set of 150+ multiple variables, denoted by $\mathrm{K}_{\mathbf{i}}$; for factorization purpose.

$$
\boldsymbol{K}_{\mathrm{i}}=\sum_{X=0}^{J} \chi_{\mathrm{ix}} \gamma_{f}+\text { Error (6) }
$$

Where, $\mathrm{K}_{\mathrm{i}}$ is a function and $\mathrm{X}$ are input variables, Error $\boldsymbol{\chi}_{\mathrm{ix}}$ are coefficients of $\gamma_{\mathrm{f}}$

\section{Model Prediction Technique}

In regression results, there are two measures considered for estimation of model fit, one is $\mathrm{R}^{2}$ and another is adjusted $\mathrm{R}^{2}$. Out of these, $\mathrm{R}$ square is usually termed as a biased measure while the adjusted $\mathrm{R}$ square is considered as the best measure for model fit estimation. Adjusted R square takes into account the change in the sample size of the datasets used for regression analysis. With the change in datasets, or the sample size, the value of $\mathrm{R}$ square gets changed, hence it is better to consider the adjusted $\mathrm{R}$ square for estimation purpose. The adjusted $\mathrm{R}$ square value is calculated when we make use of software like SAS or SPSS, for regression analysis.. The difference between the R square and adjusted R square defines the shrinkage between the two values, or the amount of change can occur in R square if the regression is applied to the population instead of the sample only. Hence, the adjusted R square is better than the $\mathrm{R}$ square, as it takes into consideration the population, not the sample. During the process of cross-validation, the value of $\mathrm{R}$ square generated from one sample is compared with the another sample of the same population. Here, the value of $R$ square of the first sample will be less than the value of $R$ square for another sample due to idiosyncratic samples. The shrinkage from the sample to the population and shrinkage between sample to sample can be different and usually high in case of cross-validation of sample to population case (Schmitt et al. 1977). It is a more accurate measure and calculated as follows:

$R^{2}=1-((\Sigma$ squared error of $Y)-(\Sigma$ squared derivation of $Y))$

$$
\mathbf{R}^{2} \text { adjusted }=1-\left(1-\mathbf{R}^{2}\right) *((\text { sample size }-1) /(\text { sample size }- \text { number of variables }-1))
$$


In this study, we have considered equation number 7 and 8 to calculate the performance comparison of proposed models developed with previous studies.

\section{Experiments}

In this section, we evaluate the FAWR technique empirically in two different experiments. The class-level metrics are used to construct a powerful model. The promise repository dataset used in this study is described in Table 1. First of all, in experiment number 1 we use exploratory factor analysis using Eq. 6 by SPSS (software V 20) to determine the influential latent or hidden variables for the robust fault prediction model development. So, 17 linear terms and 130+ interactions of linear terms implying 150+ total variables were considered for the FA of the 3 datasets considered for model development, i.e., E1, L1, and M1, and two datasets considered for model verification. From model development datasets, definite unique factors were picked based on the loadings of factors. The novel distinct factors based on independently analyzed factors for software module i.e. E1, L1, and M1 were constructed based on the average of numbers i.e. mean value. Each factor is given a factor score in each software module of the dataset. For an instance like in the E1 software dataset, on performing FA variables loaded on different factors like D1 loaded on the 10th factor, L1 loaded on 1st factor, F1 loaded on 1st factor, N1 loaded on 12th factor and so on. And after obtaining the factors from model development datasets considered the same as for model verification on the remaining 2 datasets i.e. E2 and P1 for verification.

The identification process for identifying which metric added to significant factors for $10+5$ out of $150+$ terms.

For an interpretation of illustration considering space in Table 2 i.e. "Development of factors using E1, L1, and M1 describes the identification process for identifying which metric contributes to the factors" from Sr. No. 1 to 153 display factors considering the inclusion of the coefficient value for a single variable to be 0 if it does not share the factor identified, for 3 software modules. After the identification process of factors, the important $10+5$ factors for three software modules were analyzed, to measure predictors and sharing to the factor(s). Besides, these important factors and their interaction are used to construct the robust MLR model (WI) terms.

In Table 2 (variables from sr. no. 1 to 153) highlighted, the analyzed interacting variables are presented as the names of the predictors combined by an underscore (_), for example, C1_R1 represents the predictor that is an interaction variable corresponding to $\operatorname{product}(\mathrm{X})$ of $\mathrm{C} 1$ and $\mathrm{R} 1$. For every metric, across all 3 software modules, we calculate the average (with rounding) value of the variables to which this metric contributes. And, thereafter this measured variable (metric) is combined with factors by using the rounded average value.

Table 2 Development of factors using E1, L1, and M1 describes the identification process for identifying which metric contributes to the factors.

\begin{tabular}{|c|c|c|c|c|c|c|c|c|c|c|c|c|c|c|c|c|c|}
\hline & \multicolumn{17}{|c|}{ variables } \\
\hline Sr. no. & 1 & 2 & 3 & 4 & 5 & 6 & 7 & 8 & 9 & 10 & 11 & 12 & 13 & 14 & 15 & 16 & 17 \\
\hline Software's & $\mathrm{C} 1$ & D1 & L1 & N1 & R1 & W1 & $\mathrm{F} 1$ & $\mathrm{~F} 2$ & $\mathrm{~N} 2$ & N3 & N4 & N5 & N6 & N7 & N8 & N9 & N10 \\
\hline E1 & 0 & 10 & 1 & 12 & 0 & 0 & 11 & 0 & 9 & 10 & 0 & 1 & 0 & 14 & 2 & 9 & 1 \\
\hline $\mathrm{L} 1$ & 4 & 13 & 1 & 5 & 1 & 3 & 4 & 9 & 7 & 2 & 3 & 0 & 13 & 7 & 3 & 8 & 6 \\
\hline M1 & 3 & 12 & 2 & 7 & 0 & 2 & 3 & 8 & 11 & 4 & 2 & 0 & 12 & 1 & 13 & 11 & 9 \\
\hline Mean & 2.33 & 11.6 & 1.3 & 8 & 0.3 & 1.6 & 6 & 5.6 & 9 & 5.3 & 1.6 & 0.3 & 8.3 & 7.3 & 6 & 9.3 & 5.3 \\
\hline Factor no. & 2 & 12 & 1 & 8 & 10 & 2 & 6 & 6 & 9 & 5 & 2 & 15 & 8 & 7 & 6 & 9 & 5 \\
\hline
\end{tabular}




\begin{tabular}{|c|c|c|c|c|c|c|c|c|c|c|c|c|c|}
\hline & & & & & & & variables & & & & & & \\
\hline Sr. no. & 18 & 19 & 20 & 21 & 22 & 23 & 24 & 25 & 26 & 27 & 28 & 29 & 30 \\
\hline Software's & C1_D1 & C1_L1 & C1_N1 & C1_R1 & C1_W1 & C1_F1 & C1_F2 & C1_N2 & C1_N3 & $\mathrm{C} 1 \_\mathrm{N} 4$ & C1_N5 & C1_N6 & C1_N7 \\
\hline E1 & 3 & 1 & 4 & 1 & 1 & 0 & 0 & 15 & 0 & 1 & 1 & 3 & 7 \\
\hline L1 & 4 & 1 & 5 & 1 & 1 & 4 & 1 & 1 & 2 & 1 & 1 & 4 & 1 \\
\hline M1 & 3 & 3 & 7 & 3 & 3 & 3 & 3 & 3 & 4 & 3 & 3 & 14 & 3 \\
\hline Mean & 3.3 & 1.6 & 5.3 & 1.6 & 1.6 & 2.3 & 1.3 & 6.3 & 2 & 1.6 & 1.6 & 7 & 3.6 \\
\hline Factor no. & 3 & 2 & 5 & 2 & 2 & 2 & 1 & 6 & 2 & 2 & 2 & 7 & 4 \\
\hline
\end{tabular}

\begin{tabular}{|c|c|c|c|c|c|c|c|c|c|c|c|c|c|}
\hline & & & & & & & variables & & & & & & \\
\hline Sr. no. & 31 & 32 & 33 & 34 & 35 & 36 & 37 & 38 & 39 & 40 & 41 & 42 & 43 \\
\hline E1 & 2 & 0 & 1 & 1 & 12 & 0 & 0 & 11 & 3 & 9 & 10 & 0 & 3 \\
\hline L1 & 1 & 0 & 4 & 1 & 5 & 3 & 3 & 4 & 9 & 7 & 12 & 3 & 6 \\
\hline M1 & 3 & 6 & 3 & 2 & 7 & 2 & 2 & 3 & 8 & 0 & 0 & 2 & 9 \\
\hline
\end{tabular}

\begin{tabular}{cccccccccccccc}
\hline \multicolumn{10}{c}{} & \multicolumn{10}{c}{ variables } \\
\hline Sr. no. & $\mathbf{4 4}$ & $\mathbf{4 5}$ & $\mathbf{4 6}$ & $\mathbf{4 7}$ & $\mathbf{4 8}$ & $\mathbf{4 9}$ & $\mathbf{5 0}$ & $\mathbf{5 1}$ & $\mathbf{5 2}$ & $\mathbf{5 3}$ & $\mathbf{5 4}$ & $\mathbf{5 5}$ \\
\hline Software's & D1_N6 & D1_N7 & D1_N8 & D1_N9 & D1_N10 & L1_N1 & L1_R1 & L1_W1 & L1_F1 & L1_F2 & L1_N2 & L1_N3 \\
\hline E1 & 0 & 14 & 2 & 9 & 1 & 1 & 1 & 1 & 1 & 1 & 6 \\
L1 & 13 & 7 & 3 & 8 & 6 & 1 & 1 & 1 & 1 & 1 & 1 & 2 \\
M1 & 12 & 0 & 13 & 11 & 9 & 5 & 2 & 2 & 3 & 1 & 1 & 4 & 1 \\
Mean & 8.3 & 7 & 6 & 9.3 & 5.3 & 2.3 & 1.3 & 1.3 & 1.6 & 1 & 2.6 & 3.6 \\
Factor no. & 8 & 7 & 6 & 9 & 5 & 2 & 1 & 1 & 2 & 1 & 3 \\
\hline
\end{tabular}

\begin{tabular}{|c|c|c|c|c|c|c|c|c|c|c|c|}
\hline & & & & & & variables & & & & & \\
\hline Sr. no. & 56 & 57 & 58 & 59 & 60 & 61 & 62 & 63 & 64 & 65 & 66 \\
\hline Software's & L1_N4 & L1_N5 & L1_N6 & L1_N7 & L1_N8 & L1_N10 & L1_N9 & N1_R1 & N1_W1 & N1_F1 & N1_F2 \\
\hline E1 & 1 & 1 & 7 & 0 & 1 & 1 & 6 & 13 & 13 & 4 & 4 \\
\hline L1 & 1 & 1 & 1 & 1 & 1 & 1 & 0 & 1 & 1 & 5 & 0 \\
\hline M1 & 2 & 2 & 2 & 1 & 1 & 2 & 16 & 5 & 5 & 7 & 5 \\
\hline Mean & 1.3 & 1.3 & 3.3 & 0.6 & 1 & 1.3 & 7.3 & 6.3 & 6.3 & 5.3 & 3 \\
\hline Factor no. & 1 & 1 & 3 & 1 & 1 & 1 & 7 & 6 & 6 & 5 & 3 \\
\hline
\end{tabular}

\begin{tabular}{cccccccccccccc}
\hline & \multicolumn{10}{c}{ variables } \\
\hline Sr. no. & $\mathbf{6 7}$ & $\mathbf{6 8}$ & $\mathbf{6 9}$ & $\mathbf{7 0}$ & $\mathbf{7 1}$ & $\mathbf{7 2}$ & $\mathbf{7 3}$ & $\mathbf{7 4}$ & $\mathbf{7 5}$ & $\mathbf{7 6}$ & $\mathbf{7 7}$ & $\mathbf{7 8}$ \\
\hline Software's & N1_N2 & N1_N3 & N1_N4 & N1_N5 & N1_N6 & N1_N7 & N1_N8 & N1_N9 & N1_N10 & R1_W1 R1_F1 R1_F2 \\
\hline E1 & 4 & 12 & 13 & 4 & 12 & 4 & 0 & 18 & 4 & 2 & 1 \\
L1 & 1 & 2 & 1 & 5 & 5 & 1 & 1 & 10 & 5 & 1 & 1 \\
M1 & 5 & 7 & 5 & 5 & 7 & 5 & 5 & 15 & 5 & 2 & 3 & 1 \\
Mean & 3.3 & 8 & 6.3 & 4.6 & 8 & 3.3 & 2 & 14.3 & 4.6 & 1.6 & 1.6 & 1.3 \\
Factor no. & 3 & 8 & 6 & 5 & 8 & 3 & 2 & 14 & 5 & 2 & 2 \\
\hline
\end{tabular}




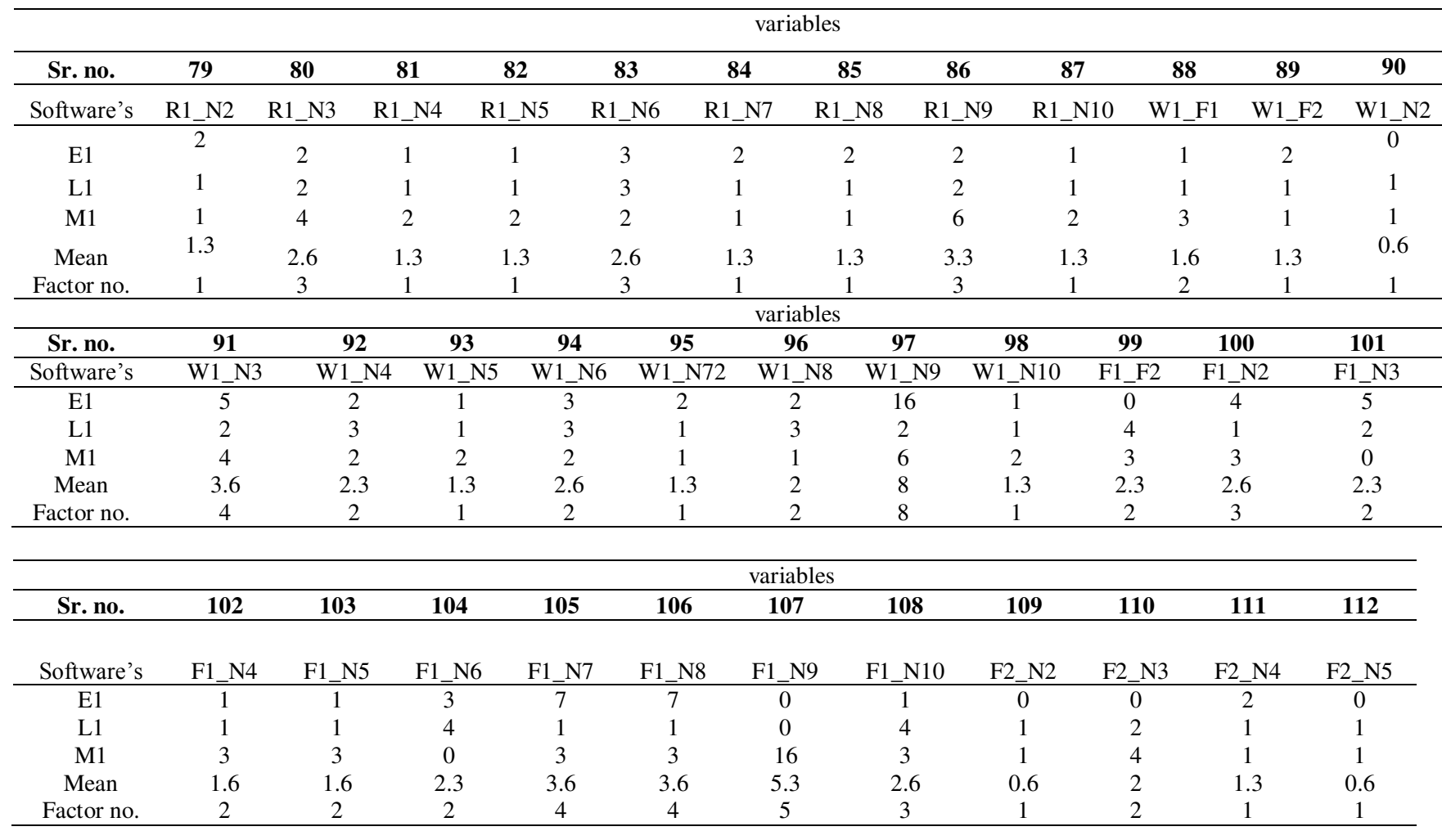

\begin{tabular}{cccccccccccc}
\hline & \multicolumn{10}{c}{ variables } \\
\hline Sr. no. & $\mathbf{1 1 3}$ & $\mathbf{1 1 4}$ & $\mathbf{1 1 5}$ & $\mathbf{1 1 6}$ & $\mathbf{1 1 7}$ & $\mathbf{1 1 8}$ & $\mathbf{1 1 9}$ & $\mathbf{1 2 0}$ & $\mathbf{1 2 1}$ & $\mathbf{1 2 2}$ & $\mathbf{1 2 3}$ \\
\hline Software's & F2_N6 & F2_N7 & F2_N8 & F2_N9 & F2_N10 & N2_N3 & N2_N4 & N2_N5 & N2_N6 & N2_N7 & N2_N8 \\
\hline E1 & 3 & 2 & 2 & 2 & 0 & 0 & 2 & 0 & 0 & 2 \\
L1 & 9 & 1 & 1 & 2 & 1 & 2 & 1 & 1 & 0 & 0 \\
M1 & 14 & 1 & 1 & 6 & 0 & 4 & 1 & 1 & 10 & 1 & 1 \\
Mean & 8.6 & 1.3 & 1.3 & 3.3 & 0.3 & 2 & 1.3 & 0.6 & 3.3 & 1 & 1.3 \\
Factor no. & 9 & 1 & 1 & 3 & 11 & 2 & 1 & 1 & 3 & 1 \\
\hline
\end{tabular}

\begin{tabular}{|c|c|c|c|c|c|c|c|c|c|c|c|c|}
\hline \multirow[b]{2}{*}{ Sr. no. } & \multicolumn{12}{|c|}{ variables } \\
\hline & 124 & 125 & 126 & 127 & 128 & 129 & 130 & 131 & 132 & 133 & 134 & 135 \\
\hline Software's & N2_N9 & N2_N10 & N3_N4 & N3_N5 & N3_N6 & N3_N7 & N3_N8 & N3_N9 & N3_N10 & N4_N5 & N4_N6 & N4_N7 \\
\hline E1 & 9 & 0 & 2 & 5 & 10 & 0 & 2 & 18 & 0 & 1 & 3 & 2 \\
\hline L1 & 0 & 1 & 2 & 2 & 12 & 2 & 2 & 2 & 2 & 1 & 3 & 1 \\
\hline M1 & 11 & 1 & 4 & 4 & 4 & 4 & 4 & 15 & 4 & 2 & 2 & 1 \\
\hline Mean & 6.6 & 0.6 & 2.6 & 3.6 & 8.6 & 2 & 2.6 & 11.6 & 2 & 1.3 & 2.6 & 1.3 \\
\hline Factor no. & 7 & 1 & 3 & 4 & 9 & 2 & 3 & 12 & 2 & 1 & 3 & 1 \\
\hline
\end{tabular}

\begin{tabular}{lccccccccccc}
\hline \multicolumn{10}{c}{ variables } \\
\hline Sr. no. & $\mathbf{1 3 6}$ & $\mathbf{1 3 7}$ & $\mathbf{1 3 8}$ & $\mathbf{1 3 9}$ & $\mathbf{1 4 0}$ & $\mathbf{1 4 1}$ & $\mathbf{1 4 2}$ & $\mathbf{1 4 3}$ & $\mathbf{1 4 4}$ & $\mathbf{1 4 5}$ & $\mathbf{1 4 6}$ \\
\hline Software's & N4_N8 & N4_N9 & N4_N10 & N5_N6 & N5_N7 & N5_N8 & N5_N9 & N5_N10 & N6_N7 & N6_N8 & N6_N9 \\
\hline E1 & 2 & 0 & 1 & 8 & 0 & 2 & 6 & 1 & 14 & 0 & 0 \\
L1 & 3 & 2 & 1 & 6 & 1 & 1 & 2 & 1 & 3 & 3 & 8 \\
M1 & 1 & 6 & 2 & 2 & 0 & 0 & 0 & 2 & 10 & 0 & 15 \\
Mean & 2 & 2.6 & 1.3 & 5.3 & 0.3 & 1 & 2.6 & 1.3 & 9 & 1 & 7.6 \\
Factor no. & 2 & 3 & 1 & 5 & 13 & 1 & 3 & 1 & 9 & 1 & 8 \\
\hline
\end{tabular}




\begin{tabular}{|c|c|c|c|c|c|c|c|}
\hline & & \multicolumn{6}{|c|}{ variables } \\
\hline Sr. no. & 147 & 148 & 149 & 150 & 151 & 152 & 153 \\
\hline Software's & N6_N10 & N7_N8 & N7_N9 & N7_N10 & N8_N9 & N8_N10 & N9_N10 \\
\hline E1 & 8 & 2 & 2 & 0 & 2 & 1 & 6 \\
\hline L1 & 6 & 1 & 2 & 1 & 0 & 1 & 2 \\
\hline M1 & 2 & 1 & 6 & 1 & 6 & 0 & 16 \\
\hline Mean & 5.3 & 1.3 & 3.3 & 0.6 & 2.6 & 0.6 & 8 \\
\hline Factor no. & 5 & 1 & 3 & 1 & 3 & 1 & 8 \\
\hline
\end{tabular}

In experiment 2, we have emphasized on two different types of regression methods i.e LR Eq. No. 1 and MLR Eq. No. 2 to analyze the modules. The other regression techniques like MLR(WI) Eq. No. 3, MLR(Self Interaction) Eq. No. 4 and SI with Square terms Eq. No. 5 to analyze the well-known data sets used in the SFP. The analysis of regression and its sub measures were performed using MATLAB R2018. Further, based on the result analysis was obtained. We have investigated the performance of the proposed method and compared it with other 4 techniques i.e. simple MLR, MLR(WI), MLR on factors, and MLR(SI). We focus on the visualization and interpretation of the multivariable data to analyze the inherent and essential property of the data sets used in the SFP. Different hidden variables WI, WOI, SI have been identified and analyzed as the input variables to predict the fault proneness. The latent variables are identified in two different ways (corresponding to two distinct experiment designs explained in this section). Therefore, about Eq. (2) of common MLR for the technique proposed Eq. (3) has been used where not only the linear dependency on the independent predictors is being observed but, also of their interactions in form of $\mathrm{Z}_{\mathrm{i}} \mathrm{Z}_{\mathrm{j}}$ (where $\mathrm{Z}_{\mathrm{i}}$ 's or $\mathrm{Z}_{\mathrm{j}}$ 's represent the unique factors) has also been analyzed for predicting the fault-proneness. Equation (4) represents the prediction of the faults (F), by the MLR(WI) model, wherein $\mathrm{Z}_{\mathrm{i}} \mathrm{Z}_{\mathrm{j}}$ is the product(*) term of two variables, where $\mathrm{Bij}$ are the coefficients of the product of two variables i.e. $Z_{\mathrm{i}}$ and $Z_{\mathrm{j}}$. For example, $Z_{1} Z_{2}$ is the product term of 2 independent factors. Alpha $\left(\alpha_{k}\right)$ are coefficients of all the square factors $Z_{k}$. All input variables were found to be selectively comprehensive and statistically good in the context of predicting faults.

Table 3 i.e. "Factors for model verification" constructed for verification of factors developed on the basis of factors developed by using 3 software modules i.e. E1, L1 and M1. However, verification of factors was applied in E2 and P1 software modules.

Table 3 Factors for model verification

\begin{tabular}{cccccccccccccccccccc}
\hline \multicolumn{10}{c}{ variables } \\
\hline Sr.no. & 1 & 2 & 3 & 4 & 5 & 6 & 7 & 8 & 9 & 10 & 11 & 12 & 13 & 14 & 15 & 16 & 17 \\
\hline Software's & C1 & D1 & L1 & N1 & R1 & W1 & F1 & F2 & N2 & N3 & N4 & N5 & N6 & N7 & N8 & N9 & N10 \\
\hline E2 & 2 & 12 & 1 & 8 & 10 & 2 & 6 & 6 & 9 & 5 & 2 & 15 & 8 & 7 & 6 & 9 & 5 \\
P1 & 2 & 12 & 1 & 8 & 10 & 2 & 6 & 6 & 9 & 5 & 2 & 15 & 8 & 7 & 6 & 9 & 5 \\
\hline
\end{tabular}

\begin{tabular}{ccccccccccccccc}
\hline & 18 & 19 & 20 & 21 & 22 & 23 & 24 & 25 & 26 & 27 & 28 & 29 & 30 \\
\hline Software's & C1_D1 & C1_L1 & C1_N1 & C1_R1 & C1_W1 & C1_F1 & C1_F2 & C1_N2 & C1_N3 & C1_N4 & C1_N5 & C1_N6 & C1_N7 \\
\hline E2 & 3 & 2 & 5 & 2 & 2 & 2 & 1 & 6 & 2 & 2 & 2 & 7 & 4 \\
P1 & 3 & 2 & 5 & 2 & 2 & 2 & 1 & 6 & 2 & 2 & 2 & 7 & 4 \\
\hline
\end{tabular}

\begin{tabular}{|c|c|c|c|c|c|c|c|c|c|c|c|c|c|}
\hline & \multicolumn{13}{|c|}{ variables } \\
\hline & 31 & 32 & 33 & 34 & 35 & 36 & 37 & 38 & 39 & 40 & 41 & 42 & 43 \\
\hline Software's & C1_N8 & C1_N9 & C1_N10 & D1_L1 & D1_N1 & D1_R1 & D1_W1 & D1_F1 & D1_F2 & D1_N2 & D1_N3 & D1_N4 & D1_N5 \\
\hline E2 & 2 & 2 & 3 & 1 & 8 & 2 & 2 & 6 & 7 & 5 & 7 & 2 & 6 \\
\hline $\mathrm{P} 1$ & 2 & 2 & 3 & 1 & 8 & 2 & 2 & 6 & 7 & 5 & 7 & 2 & 6 \\
\hline \multicolumn{14}{|c|}{ variables } \\
\hline & 44 & & 45 & 46 & 47 & 48 & 49 & 50 & 51 & 52 & 53 & 55 & \\
\hline
\end{tabular}




\begin{tabular}{ccccccccccccc}
\hline Software's & D1_N6 & D1_N7 & D1_N8 & D1_N9 & D1_N10 & L1_N1 & L1_R1 & L1_W1 & L1_F1 & L1_F2 & L1_N2 & L1_N3 \\
\hline E2 & 8 & 7 & 6 & 9 & 5 & 2 & 1 & 1 & 2 & 1 & 3 & 4 \\
P1 & 8 & 7 & 6 & 9 & 5 & 2 & 1 & 1 & 2 & 1 & 3 & 4 \\
\hline
\end{tabular}

\begin{tabular}{cccccccccccc}
\hline \multicolumn{10}{c}{ variables } \\
\hline & 56 & 57 & 58 & 59 & 60 & 61 & 62 & 63 & 64 & 65 \\
\hline Software's & L1_N4 & L1_N5 & L1_N6 & L1_N7 & L1_N8 & L1_N10 & L1_N9 & N1_R1 & N1_W1 & N1_F1 & N1_F2 \\
& & & 1 & 3 & 1 & 1 & 1 & 7 & 6 & 6 & 5 \\
E2 & 1 & 1 & 3 & 1 & 1 & 1 & 7 & 6 & 6 & 5 \\
P1 & 1 & & & & & & & & & \\
\hline
\end{tabular}

\begin{tabular}{|c|c|c|c|c|c|c|c|c|c|c|c|c|}
\hline & \multicolumn{12}{|c|}{ variables } \\
\hline & 67 & 68 & 69 & 70 & 71 & 72 & 73 & 74 & 75 & 76 & 77 & 78 \\
\hline Software' & N1_N & N1_N & N1_N & N1_N & N1_N & N1_N & N1_N & N1_N & N1_N1 & R1_W & R1_F & R1_F \\
\hline $\mathrm{s}$ & 2 & 3 & 4 & 5 & 6 & 7 & 8 & 9 & 0 & 1 & 1 & 2 \\
\hline E2 & 3 & 8 & 6 & 5 & 8 & 3 & 2 & 14 & 5 & 2 & 2 & 1 \\
\hline \multirow[t]{3}{*}{$\mathrm{P} 1$} & 3 & 8 & 6 & 5 & 8 & 3 & 2 & 14 & 5 & 2 & 2 & 1 \\
\hline & \multicolumn{12}{|c|}{ variables } \\
\hline & 79 & 80 & 81 & 82 & 83 & 84 & 85 & 86 & 87 & 88 & 89 & 90 \\
\hline Software' & R1_N & R1_N & R1_N & R1_N & R1_N & R1_N & R1_N & R1_N & R1_N1 & W1_F & W1_F & W1_N \\
\hline $\mathrm{s}$ & 2 & 3 & 4 & 5 & 6 & 7 & 8 & 9 & 0 & 1 & 2 & 2 \\
\hline E2 & 1 & 3 & 1 & 1 & 3 & 1 & 1 & 3 & 1 & 2 & 1 & 1 \\
\hline $\mathrm{P} 1$ & 1 & 3 & 1 & 1 & 3 & 1 & 1 & 3 & 1 & 2 & 1 & 1 \\
\hline
\end{tabular}

\begin{tabular}{cccccccccccc}
\hline & \multicolumn{10}{c}{ variables } & \multicolumn{10}{c}{} \\
\hline & 91 & 92 & 93 & 94 & 95 & 96 & 97 & 98 & 99 & 100 & 101 \\
\hline & W1_N3 & W1_N4 & W1_N5 & W1_N6 & W1_N72 & W1_N8 & W1_N9 & W1_N10 & F1_F2 & F1_N2 & F1_N3 \\
Software's & & & & & & & & & & & \\
\hline E2 & 4 & 2 & 1 & 2 & 1 & 2 & 8 & 1 & 2 & 3 \\
P1 & 4 & 2 & 1 & 2 & 1 & 2 & 8 & 1 & 2 & 3 & 2 \\
\hline
\end{tabular}

\begin{tabular}{cccccccccccc}
\hline \multicolumn{10}{c}{ variables } \\
\hline & 102 & 103 & 104 & 105 & 106 & 107 & 108 & 109 & 110 & 111 & 112 \\
\hline Software's & F1_N4 & F1_N5 & F1_N6 & F1_N7 & F1_N8 & F1_N9 & F1_N10 & F2_N2 & F2_N3 & F2_N4 & F2_N5 \\
\hline E2 & 2 & 2 & 2 & 4 & 4 & 5 & 3 & 1 & 2 & 1 & 1 \\
P1 & 2 & 2 & 2 & 4 & 4 & 5 & 3 & 1 & 2 & 1 & 1 \\
\hline
\end{tabular}

\begin{tabular}{cccccccccccc}
\hline \multicolumn{10}{c}{ variables } \\
\hline & 113 & 114 & 115 & 116 & 117 & 118 & 119 & 120 & 121 & 122 \\
\hline Software's & F2_N6 & F2_N7 & F2_N8 & F2_N9 & F2_N10 & N2_N3 & N2_N4 & N2_N5 & N2_N6 & N2_N7 & N2_N8 \\
\hline E2 & 9 & 1 & 1 & 3 & 11 & 2 & 1 & 1 & 3 & 1 & 1 \\
P1 & 9 & 1 & 1 & 3 & 11 & 2 & 1 & 1 & 3 & 1 & 1 \\
\hline
\end{tabular}




\begin{tabular}{|c|c|c|c|c|c|c|c|c|c|c|c|c|c|}
\hline & \multicolumn{13}{|c|}{ variables } \\
\hline & 124 & 125 & 126 & \multicolumn{2}{|l|}{127} & 128 & 129 & 130 & 131 & 132 & 133 & 134 & \multirow{2}{*}{$\frac{135}{\mathrm{~N} 4 \mathrm{N7}}$} \\
\hline Software's & N2_N9 & N2_N10 & N3_N4 & N3_N & $\mathrm{N}$ & N3_N6 & N3_N7 & N3_N8 & & N3_N10 & N4_N5 & N4_N6 & \\
\hline E2 & 7 & 1 & 3 & 4 & & 9 & 2 & 3 & 12 & 2 & 1 & 3 & 1 \\
\hline \multirow[t]{3}{*}{$\mathrm{P} 1$} & 7 & 1 & 3 & 4 & & 9 & 2 & 3 & 12 & 2 & 1 & 3 & 1 \\
\hline & \multicolumn{13}{|c|}{ variables } \\
\hline & 136 & 137 & 138 & & 139 & 12 & & 141 & 142 & 143 & 144 & 145 & 146 \\
\hline Software's & N4_N8 & N4_N9 & N4_N10 & & J5_N6 & N5 & N7 & 5_N8 & N5_N9 & N5_N10 & N6_N7 & N6_N8 & N6_N9 \\
\hline E2 & 2 & 3 & 1 & & 5 & 1 & 3 & 1 & 3 & 1 & 9 & 1 & 8 \\
\hline \multirow[t]{3}{*}{$\mathrm{P} 1$} & 2 & 3 & 1 & & 5 & 1 & 3 & 1 & 3 & 1 & 9 & 1 & 8 \\
\hline & \multicolumn{13}{|c|}{ variables } \\
\hline & & 147 & \multirow{2}{*}{\multicolumn{3}{|c|}{$\frac{148}{\text { N7_N82 }}$}} & \multicolumn{2}{|c|}{149} & \multicolumn{2}{|l|}{150} & 151 & \multicolumn{2}{|l|}{152} & 153 \\
\hline \multicolumn{2}{|l|}{ Software's } & N6_N10 & & & & N7_I & & N7_N10 & & N8_N9 & N8_N10 & & N9_N10 \\
\hline \multicolumn{2}{|l|}{ E2 } & 5 & \multicolumn{3}{|c|}{1} & \multicolumn{2}{|l|}{3} & \multicolumn{2}{|l|}{1} & 3 & \multicolumn{2}{|l|}{1} & 8 \\
\hline P1 & & 5 & \multicolumn{3}{|c|}{1} & \multicolumn{3}{|r|}{1} & & 3 & \multicolumn{2}{|l|}{1} & 8 \\
\hline
\end{tabular}


Table 4 represents the analyzed identified factor predictors i.e. B1, B2, B3, B4, B5...B15 are used to construct a powerful MLR model with interaction terms. The predictors of this model are compiled in this table.

Table 4 factor variables included in all five software modules

\begin{tabular}{|c|c|}
\hline Factor No. & Description \\
\hline B1 & $\begin{array}{l}\text { (L1+R1+W1+F2+N4+N7+C1_L1+C1_R1+C1_W1+C1_F2+C1_N4+C1_N82+D1_L1+D1_N7+L1_N1+L1_R1+L1_W1+ } \\
\text { L1_F2+L1_N2+L1_N4+L1_N5+L1_N7+L1_N8+N1_N8+R1_W1+R1_F2+R1_N2+R1_N4+R1_N5+R1_N7+R1_N8+W1 } \\
\text { _F2+W1_N2+W1_N5+W1_N7+W1_N8+F2_N2+F2_N4+F2_N5+F2_N7+F2_N8+F2_N10+N2_N4+N2_N5+N2_N7+N2 } \\
\text { _N8+N2_N10+N4_N5+N4_N7+N4_N8+N4_N10+N5_N7+N5_N8+N7_N8+N7_N10+N8_N10)/56 }\end{array}$ \\
\hline B2 & $\begin{array}{l}\left(\mathrm{C} 1+\mathrm{N} 8+\mathrm{C} 1 \_\mathrm{N} 2+\mathrm{C} 1 \_\mathrm{N} 3+\mathrm{C} 1 \_\mathrm{N} 5+\mathrm{C} 1 \_\mathrm{N} 72+\mathrm{C} 1 \_\mathrm{N} 10+\mathrm{D} 1 \_\mathrm{R} 1+\mathrm{D} 1 \_\mathrm{W} 1+\mathrm{D} 1 \_\mathrm{N} 4+\mathrm{D} 1 \_\mathrm{N} 8+\mathrm{D} 1 \text { N10+L1_F1+L1_N6+L1_N }\right. \\
\text { 10+R1_F1+R1_N10+W1_F1+W1_N4+W1_N10+F1_N3+F1_N4+F1_N5+F1_N7+F1_N8+N5_N10+N7_N9)/27 }\end{array}$ \\
\hline B3 & $\begin{array}{l}\left(\mathrm{F} 1+\mathrm{C} 1 \_\mathrm{D} 1+\mathrm{C} 1 \_\mathrm{F} 1+\mathrm{D} 1 \text { F1+D1_F2+D1_N5+R1_N6+W1_N6+F1_F2+F1_N2+F1_N6+F1_N10+N2_N6+N4_N6+N5_N }\right. \\
\text { 6+N6_N7)/16 }\end{array}$ \\
\hline B4 & $\begin{array}{l}\left(\mathrm{N} 3+\mathrm{C} 1 \_\mathrm{N} 6+\mathrm{C} 1 \_\mathrm{N} 9+\mathrm{N} 1 \_\mathrm{F} 2+\mathrm{N} 1 \_\mathrm{N} 2+\mathrm{N} 1 \_\mathrm{N} 7+\mathrm{R} 1 \_\mathrm{N} 3+\mathrm{R} 1 \_\mathrm{N} 9+\mathrm{F} 2 \_\mathrm{N} 3+\mathrm{F} 2 \_\mathrm{N} 9+\mathrm{N} 2 \_\mathrm{N} 3+\mathrm{N} 3 \_\mathrm{N} 4+\mathrm{N} 3 \_\mathrm{N} 7+\mathrm{N} 3 \_\mathrm{N} 8+\mathrm{N} 3 \_\mathrm{N}\right. \\
\left.10+\mathrm{N} 4 \_\mathrm{N} 9+\mathrm{N} 5 \_\mathrm{N} 9+\mathrm{N} 8 \_\mathrm{N} 9+\mathrm{N} 9 \_\mathrm{N} 10\right) / 19\end{array}$ \\
\hline B5 & $\left(\mathrm{C} 1 \_\mathrm{N} 1+\mathrm{L} 1 \_\mathrm{N} 3+\mathrm{L} 1 \_\mathrm{N} 9+\mathrm{N} 1\right.$ R1+N1_W1+N1_F1+N1_N4+N1_N5+N1_N10+W1_N3+W1_N9+N3_N5)/12 \\
\hline B6 & $\left(\mathrm{N} 1+\mathrm{N} 10+\mathrm{N} 6 \_\mathrm{N} 10\right) / 3$ \\
\hline B7 & $\left(\mathrm{D} 1 \_\mathrm{N} 1+\mathrm{D} 1 \_\mathrm{N} 2+\mathrm{N} 1 \_\mathrm{N} 3+\mathrm{N} 1 \_\mathrm{N} 6\right) / 4$ \\
\hline B8 & $\left(\mathrm{N} 6+\mathrm{D} 1 \_\mathrm{N} 3+\mathrm{D} 1 \_\mathrm{N} 6+\mathrm{D} 1 \_\mathrm{N} 9+\mathrm{F} 2 \_\mathrm{N} 6+\mathrm{N} 3 \_\mathrm{N} 6\right) / 6$ \\
\hline B9 & $\left(\mathrm{N} 2+\mathrm{N} 9+\mathrm{N} 2 \_\mathrm{N} 9\right) / 3$ \\
\hline $\mathrm{B} 10$ & $\left(\mathrm{D} 1+\mathrm{N} 1 \_\mathrm{N} 9\right) / 2$ \\
\hline B11 & N5 \\
\hline B12 & F1_N9 \\
\hline B13 & N6_N8 \\
\hline B14 & N3_N9 \\
\hline B15 & N6_N9 \\
\hline
\end{tabular}

Table 5 Distinct hidden factors were identified and a powerful model based on interaction terms is constructed using the important factors. A significant set of all the measured predictors sharing separately to the distinct factors If a predictor does not share with any variable, it is assumed to be a separate predictor. The powerful set of minimized terms for the $\mathrm{CK}+\mathrm{OO}$ factor variables is shown below in Table 5.

Table 5 set of all variables included in all five software modules

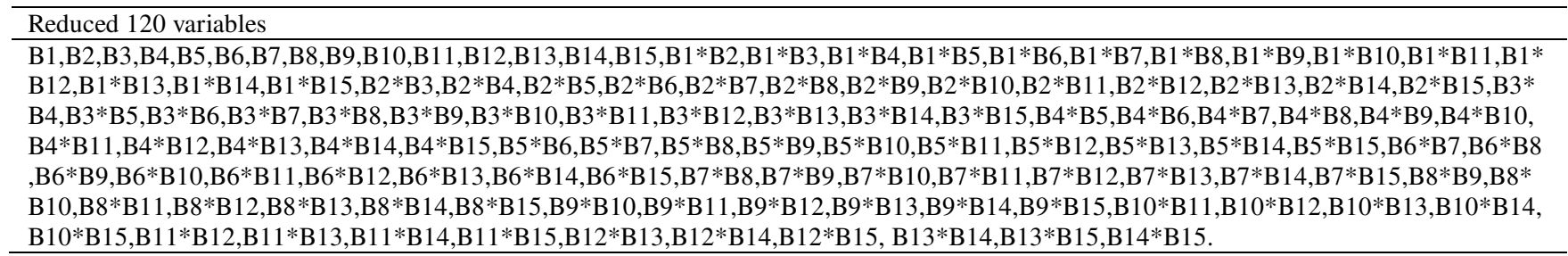




\section{Results and discussion}

The performance comparison of proposed models developed and answers to research questions are shown from Table 6 to Table 9 with figures 3 to 16. All the values of results of performance measures show the stats generated by the set of 120 and 170 terms, in both experiments taking the $\mathrm{CK}+\mathrm{OO}$ measurement attributes with their combination. The values of performance measures i.e. R Square and Adjusted R Square are significantly better among all 5 software modules as compared to the superset of 91 and 105 as suggested by (Sharma and Chandra 2019a,2020b) and 83 terms, 153 variables as suggested by (Goyal et al. 2013).

Table 6 Results of proposed model of Experiment 1 compared with (Goyal et al. 2013)

\begin{tabular}{ccccccc}
\hline Software Modules & & Our Study & \multicolumn{2}{c}{ (Goyal et al. 2013) } & \multicolumn{2}{c}{ (Goyal et al. 2013) } \\
\hline & $\mathrm{R}^{2}$ & Adj. $\mathrm{R}^{2}$ & $\mathrm{R}^{2}$ & Adj. $\mathrm{R}^{2}$ & $\mathrm{R}^{2}$ & Adj. $\mathrm{R}^{2}$ \\
\hline E1 & $\mathbf{0 . 7 7 2 4}$ & $\mathbf{0 . 7 4 1 3}$ & 0.7217 & 0.6964 & 0.7933 & 0.7558 \\
E2 & $\mathbf{0 . 9 0 5 8}$ & $\mathbf{0 . 8 5 0 9}$ & 0.8580 & 0.8089 & 0.9384 & 0.8830 \\
L1 & $\mathbf{0 . 7 0 4 3}$ & $\mathbf{0 . 6 4 2 7}$ & 0.5934 & 0.5378 & 0.6847 & 0.5948 \\
M1 & $\mathbf{0 . 4 2 6 5}$ & $\mathbf{0 . 3 8 7 3}$ & 0.4156 & 0.3883 & 0.4784 & 0.4316 \\
P1 & $\mathbf{0 . 7 3 2 1}$ & $\mathbf{0 . 7 0 9 2}$ & 0.7204 & 0.7040 & 0.7683 & 0.7419 \\
\hline
\end{tabular}

Table 7 Results comparison of the proposed model of experiment no. 1 with (Goyal et al. 2013) and (Sharma and Chandra 2019a, 2020b)

\begin{tabular}{|c|c|c|c|c|c|c|c|c|}
\hline \multirow{3}{*}{$\begin{array}{l}\text { Software } \\
\text { Modules }\end{array}$} & \multicolumn{2}{|c|}{ (Goyal et al. 2013) } & \multicolumn{2}{|c|}{ Sharma and Chandra 2019} & \multicolumn{2}{|c|}{ Sharma and Chandra 2019} & \multicolumn{2}{|c|}{ Proposed Model 1} \\
\hline & \multicolumn{2}{|c|}{83 Terms } & \multicolumn{2}{|c|}{91 Terms } & \multicolumn{2}{|c|}{105 Terms } & \multicolumn{2}{|c|}{120 Terms } \\
\hline & $\mathrm{R}^{2}$ & Adj. $R^{2}$ & $\mathrm{R}^{2}$ & Adj. $R^{2}$ & $\mathrm{R}^{2}$ & Adj. $\mathrm{R}^{2}$ & $\mathrm{R}^{2}$ & Adj. $R^{2}$ \\
\hline E1 & 0.7217 & 0.6964 & 0.7226 & 0.6950 & 0.7415 & 0.7114 & 0.7724 & 0.7413 \\
\hline E2 & 0.8580 & 0.8089 & 0.8660 & 0.8142 & 0.8722 & 0.8115 & 0.9058 & 0.8509 \\
\hline L1 & 0.5934 & 0.5378 & 0.6850 & 0.6378 & 0.6958 & 0.6418 & 0.7043 & 0.6427 \\
\hline M1 & 0.4156 & 0.3883 & 0.3655 & 0.3333 & 0.3912 & 0.3552 & 0.4265 & 0.3873 \\
\hline P1 & 0.7204 & 0.7040 & 0.6788 & 0.6582 & 0.6824 & 0.6587 & 0.7321 & 0.7092 \\
\hline
\end{tabular}

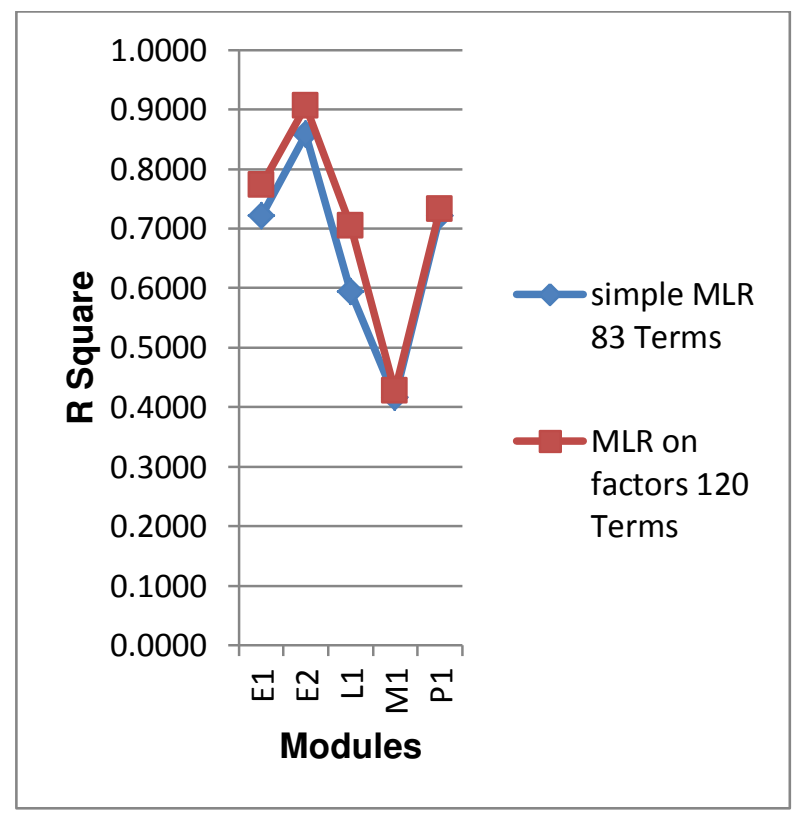

Fig.3 R square of 83 and 120 terms

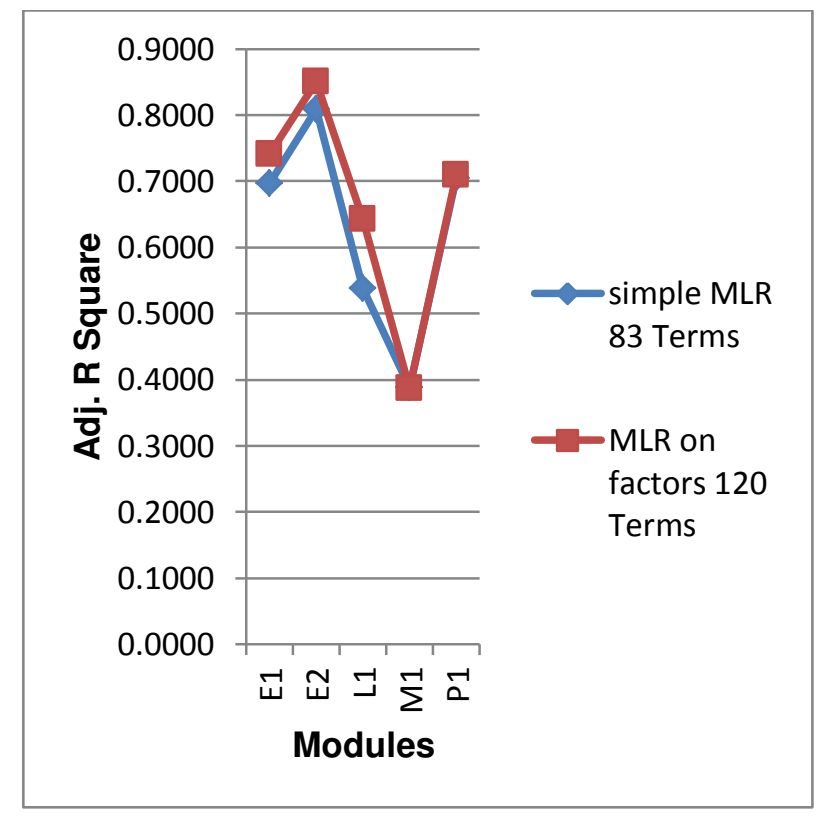

Fig.4 R square adjusted of 83 and 120 terms 


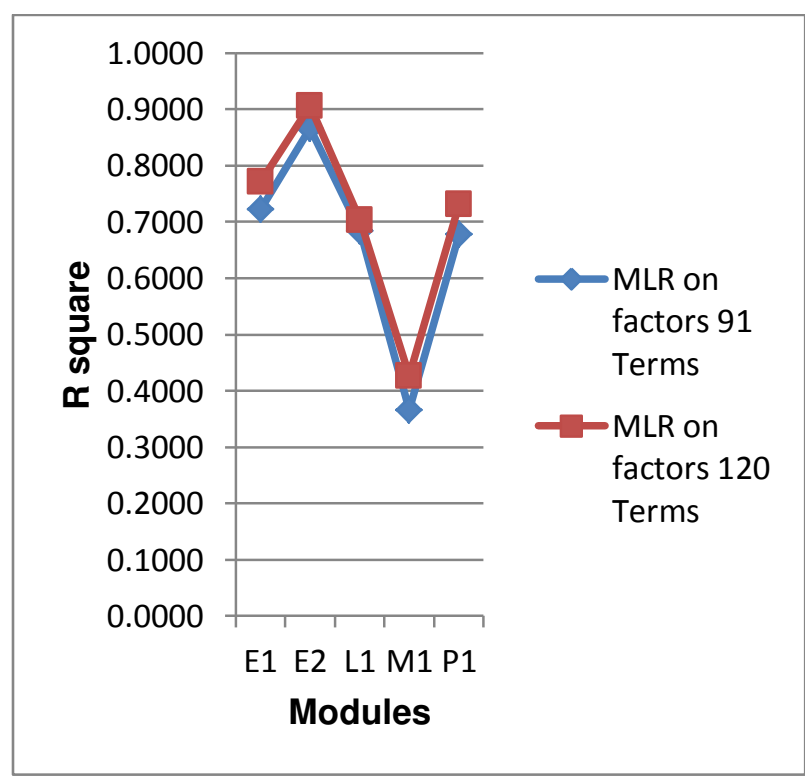

Fig.5 R square of 91 and 120 terms

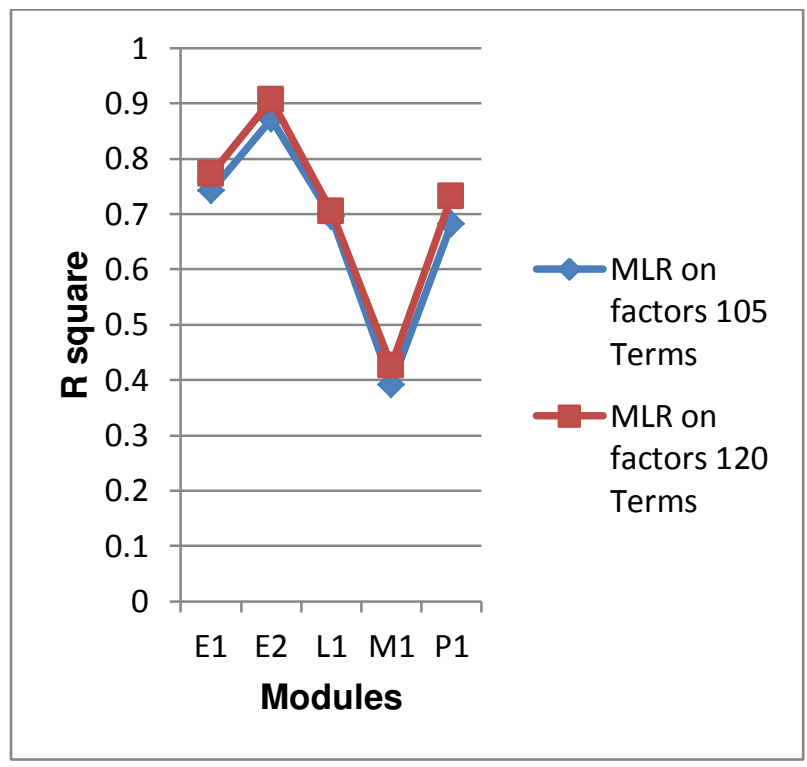

Fig.7 R square of 105 and 120 terms

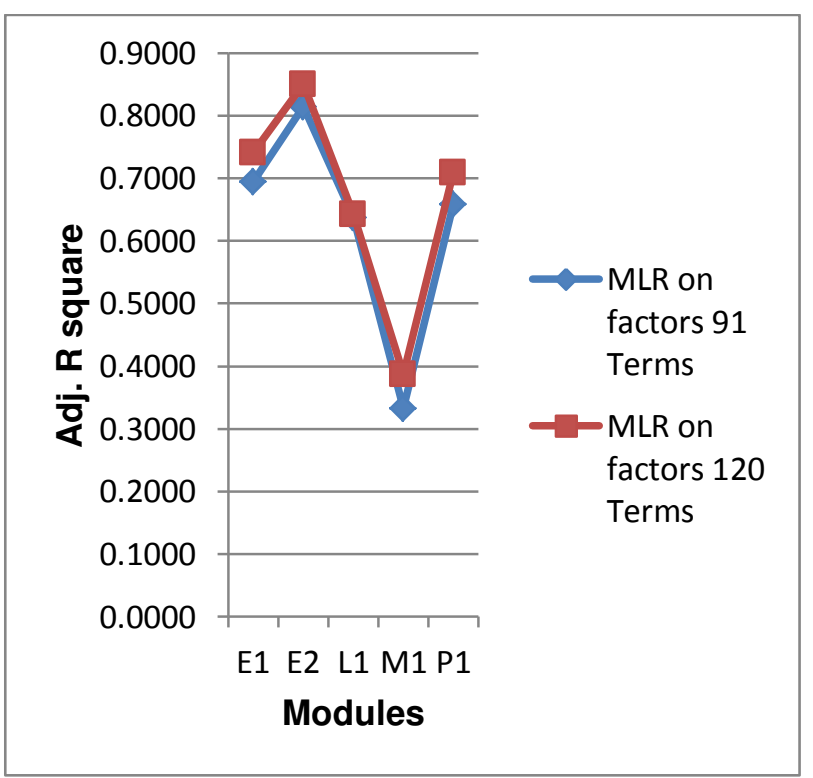

Fig.6 R square adjusted of 91 and 120 terms

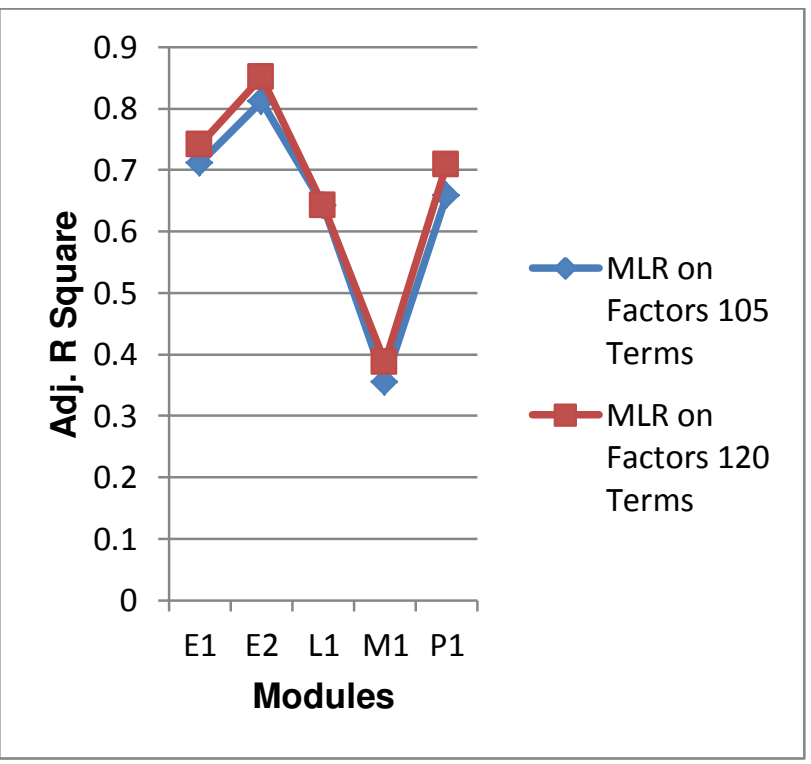

Fig.8 R square adjusted of 105 and 120 terms 
Table 8 Results comparison of the proposed model in experiment no. 2 with Goyal et al.2013

\begin{tabular}{|c|c|c|c|c|c|c|}
\hline \multirow[t]{3}{*}{ Software Modules } & \multicolumn{2}{|c|}{ Goyal et al.2013 } & \multicolumn{2}{|c|}{ Goyal et al.2013 } & \multicolumn{2}{|c|}{ Proposed Model 2} \\
\hline & \multicolumn{2}{|c|}{83 Terms } & \multicolumn{2}{|c|}{153 Terms } & \multicolumn{2}{|c|}{170 Terms } \\
\hline & $\mathrm{R}^{2}$ & Adj. $\mathrm{R}^{2}$ & $\mathrm{R}^{2}$ & Adj. $R^{2}$ & $\mathrm{R}^{2}$ & Adj. $R^{2}$ \\
\hline E1 & 0.7217 & 0.6964 & 0.7933 & 0.7558 & 0.8173 & 0.7789 \\
\hline E2 & 0.8580 & 0.8089 & 0.9384 & 0.8830 & 0.9501 & 0.8947 \\
\hline L1 & 0.5934 & 0.5378 & 0.6847 & 0.5948 & 0.7000 & 0.6019 \\
\hline M1 & 0.4156 & 0.3883 & 0.4784 & 0.4316 & 0.4881 & 0.4366 \\
\hline P1 & 0.7204 & 0.7040 & 0.7683 & 0.7419 & 0.7804 & 0.7522 \\
\hline
\end{tabular}

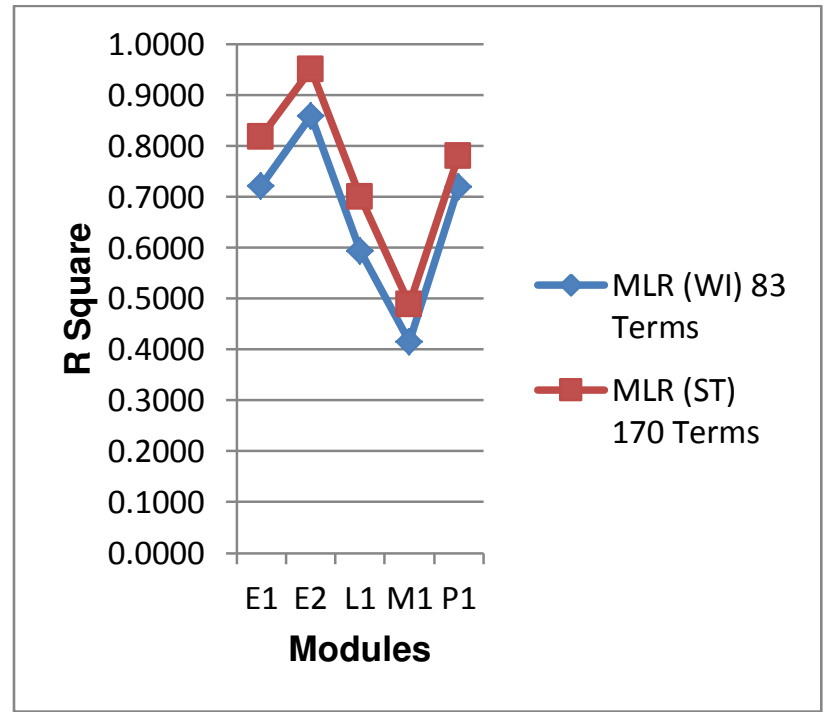

Fig.9 R square of 83 and 170 terms

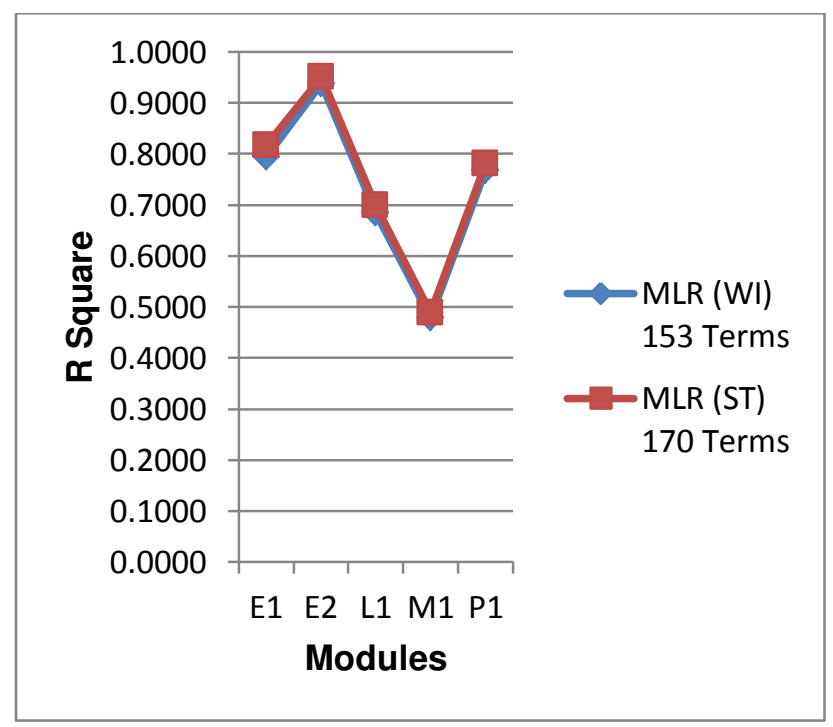

Fig.11 R square of 153 and 170 terms

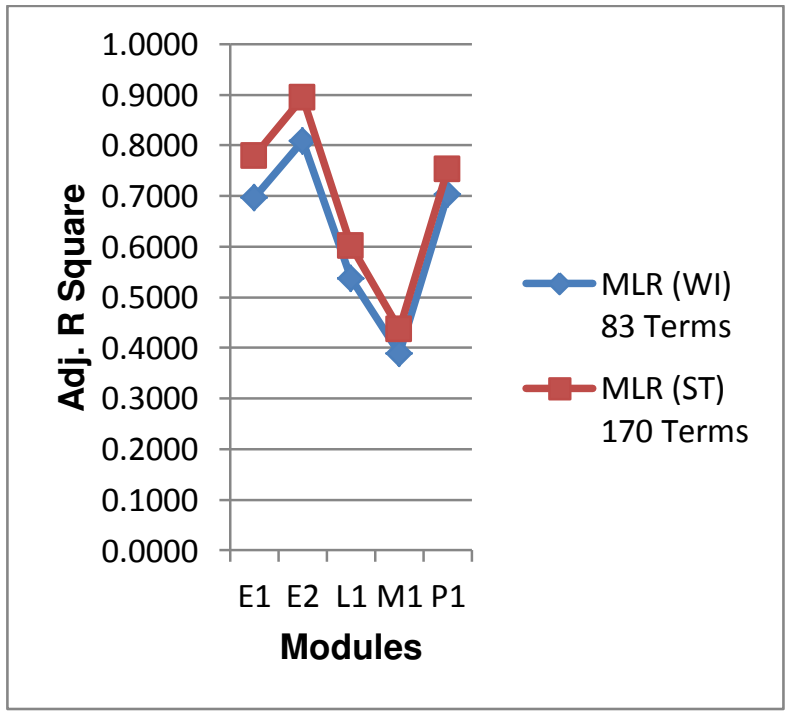

Fig.10 R square adjusted of 83 and 170 terms

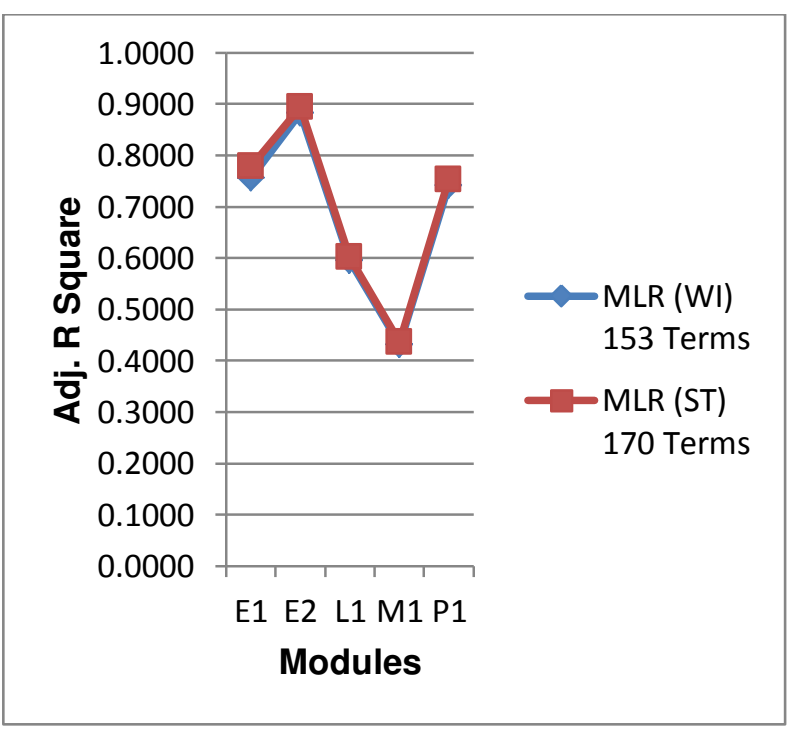

Fig.12 R square adjusted of 153 and 170 terms 
Table 9 Results comparison of the proposed model in experiment no. 2 with Sharma and Chandra 2019a,2020b

\begin{tabular}{ccccccc}
\hline \multirow{2}{*}{$\begin{array}{c}\text { Software } \\
\text { Modules }\end{array}$} & \multicolumn{2}{c}{$\begin{array}{c}\text { Sharma and Chandra 2019 } \\
\text { 91 Terms }\end{array}$} & \multicolumn{2}{c}{ Sharma and Chandra 2020 } & \multicolumn{2}{c}{$\begin{array}{c}\text { Proposed Model 2 } \\
\text { 170 Terms }\end{array}$} \\
\cline { 2 - 7 } & $\mathrm{R}^{2}$ & Adj. $\mathrm{R}^{2}$ & $\mathrm{R}^{2}$ & Adj. $\mathrm{R}^{2}$ & $\mathbf{R}^{\mathbf{2}}$ & Adj. $\mathbf{R}^{\mathbf{2}}$ \\
\hline E1 & 0.7226 & 0.6950 & 0.7415 & 0.7114 & $\mathbf{0 . 8 1 7 3}$ & $\mathbf{0 . 7 7 8 9}$ \\
E2 & 0.8660 & 0.8142 & 0.8722 & 0.8115 & $\mathbf{0 . 9 5 0 1}$ & $\mathbf{0 . 8 9 4 7}$ \\
L1 & 0.6850 & 0.6378 & 0.6958 & 0.6418 & $\mathbf{0 . 7 0 0 0}$ & $\mathbf{0 . 6 0 1 9}$ \\
M1 & 0.3655 & 0.3333 & 0.3912 & 0.3552 & $\mathbf{0 . 4 8 8 1}$ & $\mathbf{0 . 4 3 6 6}$ \\
P1 & 0.6788 & 0.6582 & 0.6824 & 0.6587 & $\mathbf{0 . 7 8 0 4}$ & $\mathbf{0 . 7 5 2 2}$ \\
\hline
\end{tabular}

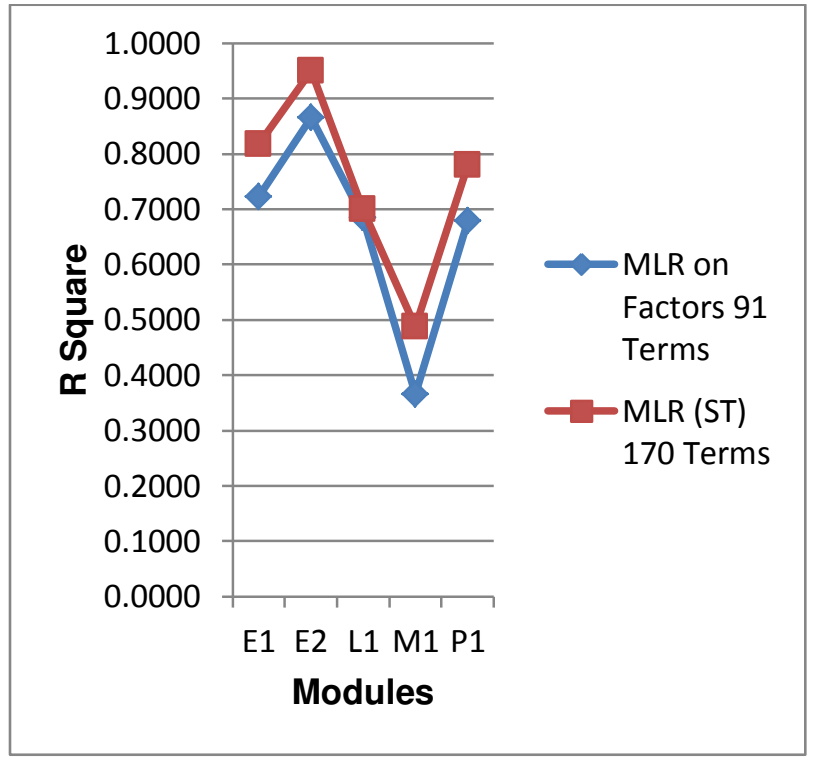

Fig.13 R square of 91 and 170 terms

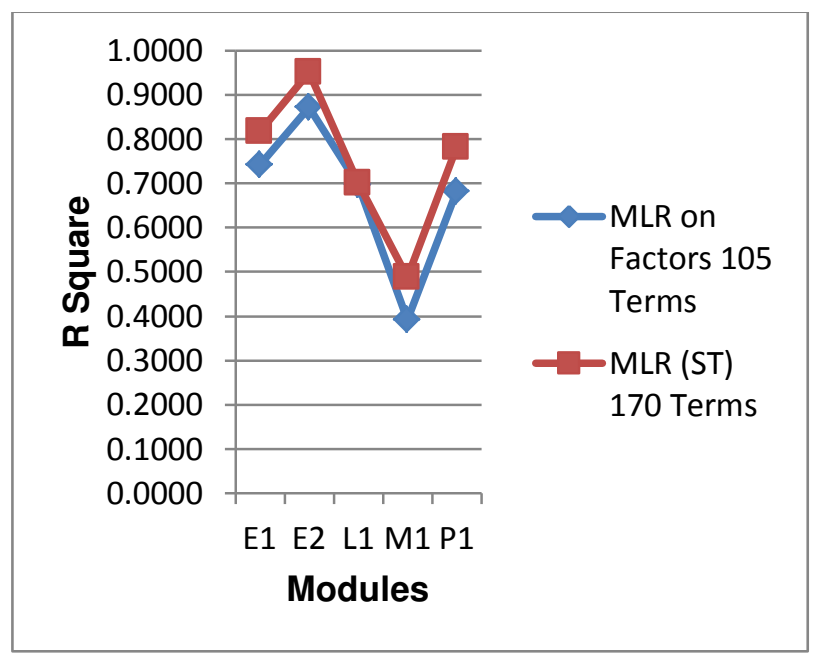

Fig.15 R square of 105 and 170 terms

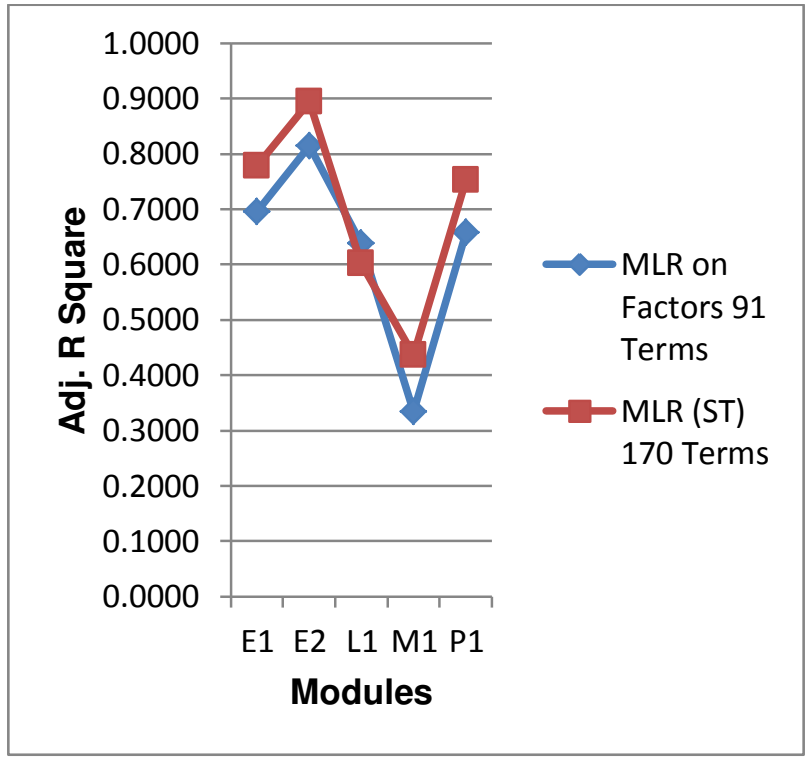

Fig.14 R square adjusted of 91 and 170 terms

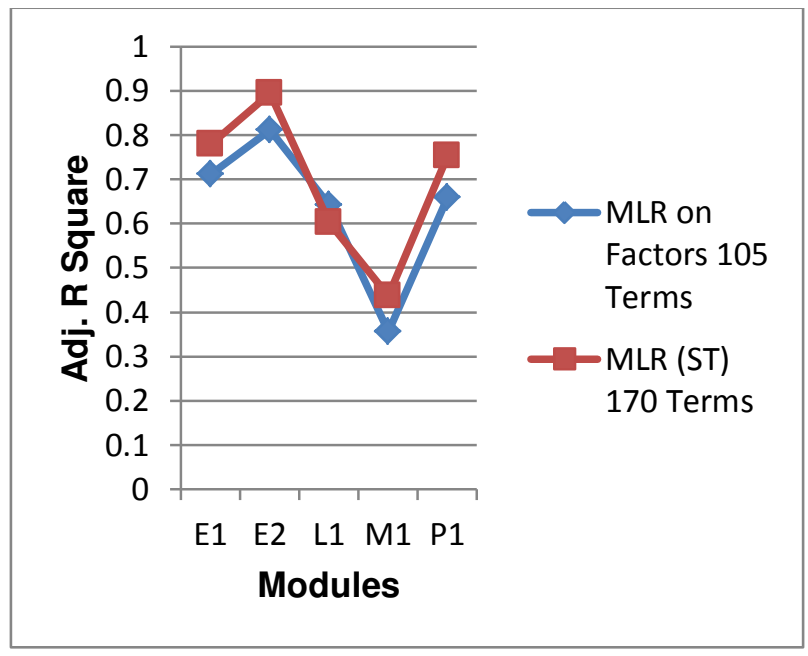

Fig.16 R square adjusted of 105 and 170 terms 


\section{Threats to validity}

This section explains the possible threats to the reliability and validity of fault proneness. The objective of this study was to analyze the reliability and fault prediction of different software modules based on FAWR techniques. The scope of this research is restricted to the effect of linear terms with interaction and square of linear terms and their comparison with factor variables in the context of simple linear regression. Soft computing techniques have other well-constructed analytical methods of classification and feature selection, which are beyond the scope of this research work. The selection criteria of the comparative studies based on reliability model assessment questions were done independently so that biases can be avoided. To predict the faults and various models development, the data were extracted from recent studies (D'Ambros et al. 2010, 2012; Goyal et al. 2013; Sharma and Chandra 2019a, 2020b). The bug prediction data were taken from an open-source promise repository for experiments in this research work. However, the methods were performed under varied experimental design set up (selection of input variables, validation method used, performance measures used, etc.). Thus, the performance measures of the prediction of different predictors using the proposed technique for software developed in the IT sector might be different than the existing results. Further, the outcome obtained for the proposed model and methods proposed are encouraging, but the empirical analysis is needed before the goodness of fit of the methods that can be generalized outside this area, in particular, different modules of the software. Also, in this study, two different experiments were performed using FAWR models for bug prediction, and the performance may vary for latent variables and square terms count in proposed models, which could be a possible threat to the study. Another threat might be the coding. The strengths and weaknesses presented are based on the opinion of the respective authors.

\section{Conclusion and outlook}

One of the vital objectives of this research work was to motivate researchers and software scientists who can improve empirical analyses to develop good software quality models by taking the combinations including interactions of factor variables and square terms into consideration. The assumption of error-free metrics requires further evaluation. Moreover, in empirical analysis different metrics affects one another. Practitioners use process or product metrics in the development of software fault prediction models. However, these measurement variables are related and when taken in interaction (product terms), they may lead to a better effect on the predictive capability of the prediction model. Results derived from the study indicate that the authenticity of the software fault proneness is highly dependent on the interacting variables. And the results showed that the regression analysis on interacting square terms and factor variables are better than the stepwise MLR. The results obtained from the set of 120-factor variables are in well agreement with the results of (Goyal et al. 2013; Sharma and Chandra 2019a, 2020b). Similarly, the comparisons of results show the performance of model fitting in terms of R-square and adj. R-square tables 9 and 10 with figures 17 and 18 in comparison with each other. However, results obtained by the set of 120 and 170 (square terms) variables are comparatively better in both experiments across all five software modules. Therefore, we conclude that after the reduction of terms, the values of results are also significantly better. Therefore, square factor and factor analysis with regression is a very powerful technique for the prediction of faults in software. The processes and techniques presented in this study can be utilized to develop good quality software models including the important characteristics of software like testing the functionality, efficiency, serviceability, dependability, authenticity and maintainability.

Table 10 Results comparison of R square of the proposed model in experiment no. 1 and 2 with Sharma and Chandra 2019a, 2020b and Goyal et al. 2013

\begin{tabular}{ccccccc}
\hline \multirow{2}{*}{$\begin{array}{c}\text { Software } \\
\text { Modules }\end{array}$} & \multicolumn{2}{c}{ Sharma an d Chandra 2019 Sharma and Chandra 2020 } & Goyal et al.2013 & Goyal et al.2013 & $\begin{array}{l}\text { Proposed } \\
\text { Model 1 }\end{array}$ & $\begin{array}{c}\text { Proposed } \\
\text { Model 2 }\end{array}$ \\
\cline { 2 - 6 } & $\mathrm{R}^{2}$ & $\mathrm{R}^{2}$ & $\mathrm{R}^{2}$ & $\mathrm{R}^{2}$ & $\mathrm{R}^{2}$ \\
\hline E1 & 0.722 & 0.741 & 0.7217 & 0.7933 & $\mathbf{0 . 7 7 2 4}$ & $\mathbf{0 . 8 1 7 3}$ \\
E2 & 0.866 & 0.872 & 0.8580 & 0.9384 & $\mathbf{0 . 9 0 5 8}$ & $\mathbf{0 . 9 5 0 1}$ \\
L1 & 0.685 & 0.695 & 0.5934 & 0.6847 & $\mathbf{0 . 7 0 4 3}$ & $\mathbf{0 . 7 0 0 0}$ \\
M1 & 0.365 & 0.391 & 0.4156 & 0.4784 & $\mathbf{0 . 4 2 6 5}$ & $\mathbf{0 . 4 8 8 1}$ \\
P1 & 0.678 & 0.682 & 0.7204 & 0.7683 & $\mathbf{0 . 7 3 2 1}$ & $\mathbf{0 . 7 8 0 4}$ \\
\hline
\end{tabular}




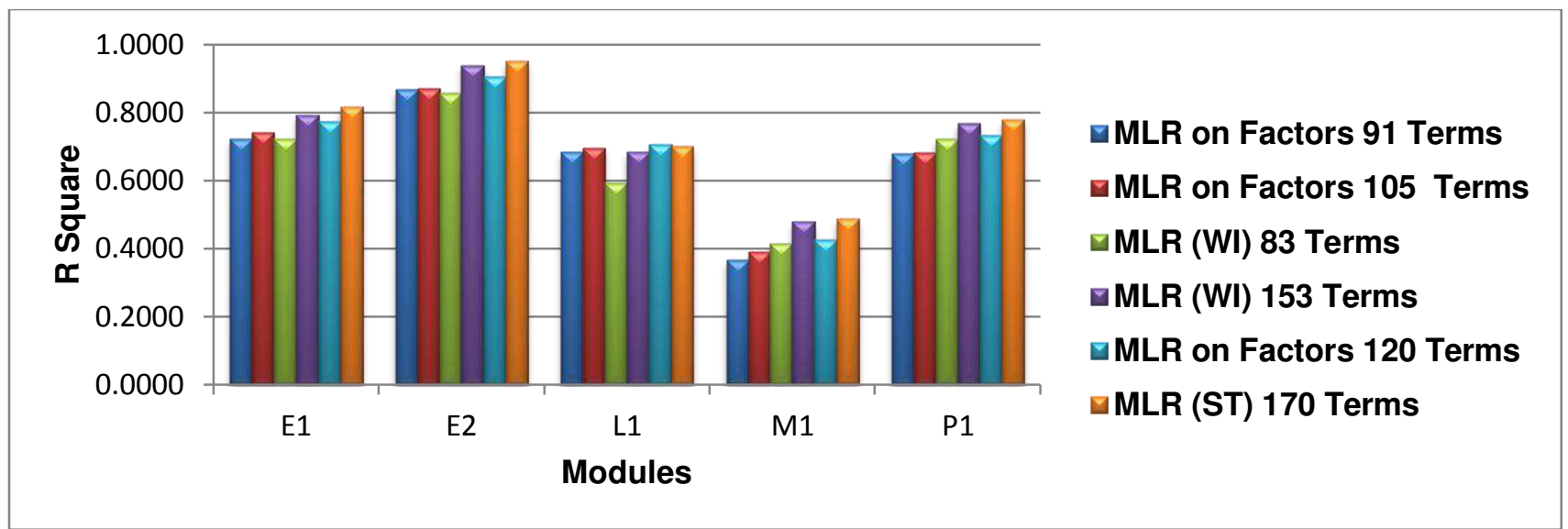

Fig.17 R square of 91, 105, 83, 153, 120 and 170 terms

Table 11 Results comparison of R square adjusted of proposed models in experiment no. 1 and 2 with Sharma et and Chandra 2019a, 2020b and Goyal et al.2013

\begin{tabular}{ccccccc}
\hline \multirow{2}{*}{$\begin{array}{c}\text { Software } \\
\text { Modules }\end{array}$} & $\begin{array}{c}\text { Sharma and Chandra } \\
\text { 2019 }\end{array}$ & $\begin{array}{c}\text { Sharma and Chandra } \\
\text { Adj. } \mathrm{R}^{2}\end{array}$ & Goyal et al.2013 & Goyal et al.2013 & Proposed Model 1 Proposed Model 2 \\
\cline { 2 - 6 } & 0.695 & Adj. $\mathrm{R}^{2}$ & Adj. $\mathrm{R}^{2}$ & Adj. $\mathrm{R}^{2}$ & ${\text { Adj. } \mathrm{R}^{2}}$ & Adj. $^{2}$ \\
\cline { 2 - 7 } E1 & 0.814 & 0.711 & 0.696 & 0.755 & $\mathbf{0 . 7 4 1 3}$ & $\mathbf{0 . 7 7 8 9}$ \\
E2 & 0.637 & 0.641 & 0.808 & 0.883 & $\mathbf{0 . 8 5 0 9}$ & $\mathbf{0 . 8 9 4 7}$ \\
L1 & 0.333 & 0.355 & 0.537 & 0.594 & $\mathbf{0 . 6 4 2 7}$ & $\mathbf{0 . 6 0 1 9}$ \\
M1 & 0.658 & 0.658 & 0.388 & 0.431 & $\mathbf{0 . 3 8 7 3}$ & $\mathbf{0 . 4 3 6 6}$ \\
P1 & & 0.704 & 0.741 & $\mathbf{0 . 7 0 9 2}$ & $\mathbf{0 . 7 5 2 2}$ \\
\hline
\end{tabular}

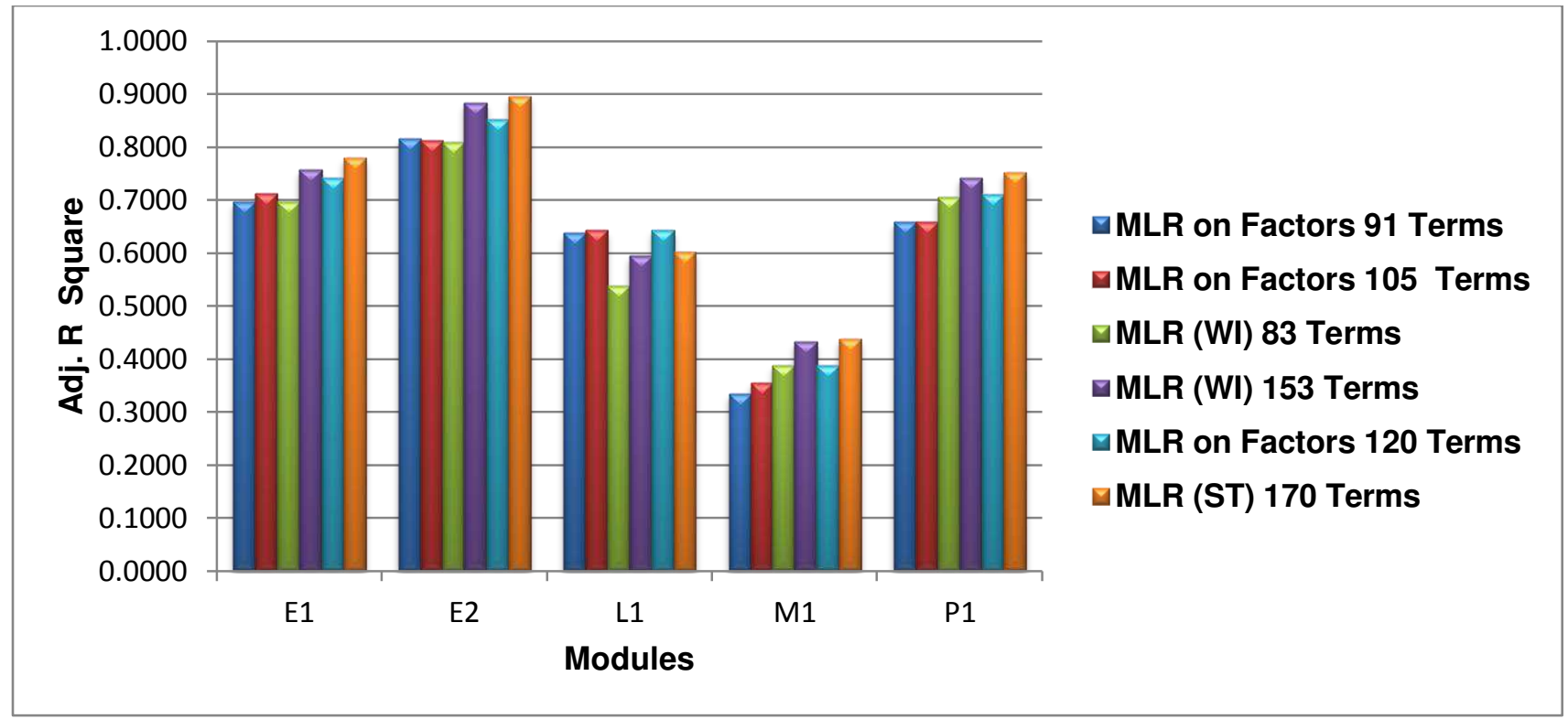

Fig.18 R square adjusted of 91, 105, 83, 153, 120 and 170 terms 


\section{Declarations}

- The authors have no relevant financial or non-financial interests to disclose.

- The authors have no conflicts of interest to declare that are relevant to the content of this article.

- All authors certify that they have no affiliations with or involvement in any organization or entity with any financial interest or non-financial interest in the subject matter or materials discussed in this manuscript.

- The authors have no financial or proprietary interests in any material discussed in this article.

\section{References}

Aggarwal, K. K., Singh, Y., Kaur, A., \& Malhotra, R. (2009). Empirical analysis for investigating the effect of object-oriented metrics on fault proneness: a replicated case study. Software Process: Improvement and Practice, 14(1), 39-62. https://doi.org/10.1002/spip.389

Aiken, L. S., West, S. G., Pitts, S. C., Baraldi, A. N., \& Wurpts, I. C. (2012). Multiple Linear Regression. In I. Weiner (Ed.), Handbook of Psychology, Second Edition (p. hop202018). Hoboken, NJ, USA: John Wiley \& Sons, Inc. https://doi.org/10.1002/9781118133880.hop202018

Al Dallal, J. (2011). Improving the applicability of object-oriented class cohesion metrics. Information and Software Technology, 53(9), 914-928. https://doi.org/10.1016/j.infsof.2011.03.004

Al Dallal, J. (2012a). Fault prediction and the discriminative powers of connectivity-based object-oriented class cohesion metrics. Information and Software Technology, 54(4), 396-416. https://doi.org/10.1016/j.infsof.2011.11.007

Al Dallal, J. (2012b). Fault prediction and the discriminative powers of connectivity-based object-oriented class cohesion metrics. Information and Software Technology, 54(4), 396-416. https://doi.org/10.1016/j.infsof.2011.11.007

Al Dallal, J. (2013). Object-oriented class maintainability prediction using internal quality attributes. Information and Software Technology, 55(11), 2028-2048. https://doi.org/10.1016/j.infsof.2013.07.005

Al Dallal, J. (2018). Predicting Fault-Proneness of Reused Object-Oriented Classes in Software Post-Releases. Arabian Journal for Science and Engineering, 43(12), 7153-7166. https://doi.org/10.1007/s13369-017-3012-2

Al Dallal, J., \& Morasca, S. (2014). Predicting object-oriented class reuse-proneness using internal quality attributes. Empirical Software Engineering, 19(4), 775-821. https://doi.org/10.1007/s10664-012-9239-3

Arar, Ö. F., \& Ayan, K. (2015). Software defect prediction using cost-sensitive neural network. Applied Soft Computing, 33, 263-277. https://doi.org/10.1016/j.asoc.2015.04.045

Basili, V. R., Briand, L. C., \& Melo, W. L. (1996). A validation of object-oriented design metrics as quality indicators. IEEE Transactions on Software Engineering, 22(10), 751-761. https://doi.org/10.1109/32.544352

Briand, L. C., Wüst, J., \& Lounis, H. (2001). Replicated Case Studies for Investigating Quality Factors in ObjectOriented Designs. Empirical Software Engineering, 6(1), 11-58. https://doi.org/10.1023/A:1009815306478

Browne, M. W. (1969). Fitting the factor analysis model. Psychometrika, 34(3), 375-394. https://doi.org/10.1007/BF02289365

Chandrasekaran, M., Muralidhar, M., Krishna, C. M., \& Dixit, U. S. (2010). Application of soft computing techniques in machining performance prediction and optimization: a literature review. The International Journal of Advanced Manufacturing Technology, 46(5-8), 445-464. https://doi.org/10.1007/s00170-009-2104-x

Chatterjee, S., Nigam, S., \& Roy, A. (2017). Software fault prediction using neuro-fuzzy network and evolutionary learning approach. Neural Computing and Applications, 28(S1), 1221-1231. https://doi.org/10.1007/s00521-016$2437-y$

Chatterjee, Subhashis, \& Roy, A. (2015). Novel Algorithms for Web Software Fault Prediction. Quality and Reliability Engineering International, 31(8), 1517-1535. https://doi.org/10.1002/qre.1687

Chatterjee, Subhashis, \& Shukla, A. (2016). Modeling and Analysis of Software Fault Detection and Correction Process Through Weibull-Type Fault Reduction Factor, Change Point and Imperfect Debugging. Arabian Journal for Science and Engineering, 41(12), 5009-5025. https://doi.org/10.1007/s13369-016-2189-0

Chatterjee, S., Maji, B. A new fuzzy rule based algorithm for estimating software faults in early phase of development. Soft Comput 20, 4023-4035 (2016). https://doi.org/10.1007/s00500-015-1738-x

Chaturvedi, D. K. (2008). Soft Computing (Vol. 103). Berlin, Heidelberg: Springer Berlin Heidelberg. https://doi.org/10.1007/978-3-540-77481-5 
Chiang, M.-C., Huang, C.-Y., Wu, C.-Y., \& Tsai, C.-Y. (2020). Analysis of a Fault-Tolerant Framework for Reliability Prediction of Service-Oriented Architecture Systems. IEEE Transactions on Reliability, 1-36. https://doi.org/10.1109/TR.2020.2968884

Chidamber, S. R., \& Kemerer, C. F. (1994). A metrics suite for object oriented design. IEEE Transactions on Software Engineering, 20(6), 476-493. https://doi.org/10.1109/32.295895

Chinna Gounder Dhanajayan, R., \& Appavu Pillai, S. (2017). SLMBC: spiral life cycle model-based Bayesian classification technique for efficient software fault prediction and classification. Soft Computing, 21(2), 403-415. https://doi.org/10.1007/s00500-016-2316-6

Cohen. (2013). Applied Multiple Regression/Correlation Analysis for the Behavioral Sciences (3rd ed.). Routledge. https://doi.org/10.4324/9780203774441

Colanzi, T. E., Assunção, W. K. G., Vergilio, S. R., Farah, P. R., \& Guizzo, G. (2020). The Symposium on SearchBased Software Engineering: Past, Present and Future. Information and Software Technology, 127, 106372. https://doi.org/10.1016/j.infsof.2020.106372

Dagpinar, M., \& Jahnke, J. H. (2003). Predicting maintainability with object-oriented metrics -an empirical comparison. In 10th Working Conference on Reverse Engineering, 2003. WCRE 2003. Proceedings. (pp. 155164). Presented at the 10th Working Conference on Reverse Engineering, 2003. WCRE 2003., Victoria, BC, Canada: IEEE. https://doi.org/10.1109/WCRE.2003.1287246

Dejaeger, K., Verbraken, T., \& Baesens, B. (2013). Toward Comprehensible Software Fault Prediction Models Using Bayesian Network Classifiers. IEEE Transactions on Software Engineering, 39(2), 237-257. https://doi.org/10.1109/TSE.2012.20

Di Nucci, D., Palomba, F., De Rosa, G., Bavota, G., Oliveto, R., \& De Lucia, A. (2018). A Developer Centered Bug Prediction Model. IEEE Transactions on Software Engineering, 44(1), 5-24. https://doi.org/10.1109/TSE.2017.2659747

Erturk, E., \& Akcapinar Sezer, E. (2016). Iterative software fault prediction with a hybrid approach. Applied Soft Computing, 49, 1020-1033. https://doi.org/10.1016/j.asoc.2016.08.025

Erturk, E., \& Sezer, E. A. (2015). A comparison of some soft computing methods for software fault prediction. Expert Systems with Applications, 42(4), 1872-1879. https://doi.org/10.1016/j.eswa.2014.10.025

Goel, A. L., \& Okumoto, K. (1979). Time-Dependent Error-Detection Rate Model for Software Reliability and Other Performance Measures. IEEE Transactions on Reliability, $R$-28(3), 206-211. https://doi.org/10.1109/TR.1979.5220566

Goyal, R., Chandra, P., \& Singh, Y. (2013). Identifying influential metrics in the combined metrics approach of fault prediction. SpringerPlus, 2(1), 627. https://doi.org/10.1186/2193-1801-2-627

Gyimothy, T., Ferenc, R., \& Siket, I. (2005). Empirical validation of object-oriented metrics on open source software for fault prediction. IEEE Transactions on Software Engineering, 31(10), 897-910. https://doi.org/10.1109/TSE.2005.112

Harman, G. H., \& Chomsky, N. (1967). Psychological Aspects of the Theory of Syntax. The Journal of Philosophy, 64(2), 75. https://doi.org/10.2307/2023773

Hassan, A. E. (2009). Predicting faults using the complexity of code changes. In 2009 IEEE 31st International Conference on Software Engineering (pp. 78-88). Presented at the 2009 IEEE 31st International Conference on Software Engineering, Vancouver, BC, Canada: IEEE. https://doi.org/10.1109/ICSE.2009.5070510

Huang, C.-Y., \& Lyu, M. R. (2005). Optimal Testing Resource Allocation, and Sensitivity Analysis in Software Development. IEEE Transactions on Reliability, 54(4), 592-603. https://doi.org/10.1109/TR.2005.858099

Jabangwe, R., Börstler, J., Šmite, D., \& Wohlin, C. (2015). Empirical evidence on the link between object-oriented measures and external quality attributes: a systematic literature review. Empirical Software Engineering, 20(3), 640-693. https://doi.org/10.1007/s10664-013-9291-7

Kapur, P. K., Pham, H., Anand, S., \& Yadav, K. (2011). A Unified Approach for Developing Software Reliability Growth Models in the Presence of Imperfect Debugging and Error Generation. IEEE Transactions on Reliability, 60(1), 331-340. https://doi.org/10.1109/TR.2010.2103590

Karnavas, W. J., Sanchez, P. J., \& Bahill, A. T. (1993). Sensitivity analyses of continuous and discrete systems in the time and frequency domains. IEEE Transactions on Systems, Man, and Cybernetics, 23(2), 488-501. https://doi.org/10.1109/21.229461

Killick, R., \& Eckley, I. A. (2014). An R package for change point analysis. Journal of Statistical Software, 58(3). https://doi.org/10.18637/jss.v058.i03

Kitchenham, B. A., Hughes, R. T., \& Linkman, S. G. (2001). Modeling software measurement data. IEEE Transactions on Software Engineering, 27(9), 788-804. https://doi.org/10.1109/32.950316 
Kosker, Y., Turhan, B., \& Bener, A. (2009). An expert system for determining candidate software classes for refactoring. Expert Systems with Applications, 36(6), 10000-10003. https://doi.org/10.1016/j.eswa.2008.12.066

Lawley, D. N., \& Maxwell, A. E. (1973). Regression ana factor analysis. Biometrika, 60(2), 331-338. https://doi.org/10.1093/biomet/60.2.331

Liang Yin, \& Trivedi, K. S. (1999). Confidence interval estimation of NHPP-based software reliability models. In Proceedings 10th International Symposium on Software Reliability Engineering (Cat. No.PR00443) (pp. 6-11). Presented at the 10th International Symposium on Software Reliability Engineering, Boca Raton, FL, USA: IEEE Comput. Soc. https://doi.org/10.1109/ISSRE.1999.809305

Lo, J.-H., Huang, C.-Y., Chen, I.-Y., Kuo, S.-Y., \& Lyu, M. R. (2005). Reliability assessment and sensitivity analysis of software reliability growth modeling based on software module structure. Journal of Systems and Software, 76(1), 3-13. https://doi.org/10.1016/j.jss.2004.06.025

Mockus, A., \& Weiss, D. M. (2002). Predicting risk of software changes. Bell Labs Technical Journal, 5(2), 169180. https://doi.org/10.1002/bltj.2229

Mohanty, R., Ravi, V., \& Patra, M. R. (2010). The application of intelligent and soft-computing techniques to software engineering problems: a review. International Journal of Information and Decision Sciences, 2(3), 233. https://doi.org/10.1504/IJIDS.2010.033450

Olague, H. M., Etzkorn, L. H., Gholston, S., \& Quattlebaum, S. (2007). Empirical Validation of Three Software Metrics Suites to Predict Fault-Proneness of Object-Oriented Classes Developed Using Highly Iterative or Agile Software Development Processes. IEEE Transactions on Software Engineering, 33(6), 402-419. https://doi.org/10.1109/TSE.2007.1015

Panichella, A., Alexandru, C. V., Panichella, S., Bacchelli, A., \& Gall, H. C. (2016). A Search-based Training Algorithm for Cost-aware Defect Prediction. In Proceedings of the Genetic and Evolutionary Computation Conference 2016 (pp. 1077-1084). Presented at the GECCO '16: Genetic and Evolutionary Computation Conference, Denver Colorado USA: ACM. https://doi.org/10.1145/2908812.2908938

Pillai, K., \& Sukumaran Nair, V. S. (1997). A model for software development effort and cost estimation. IEEE Transactions on Software Engineering, 23(8), 485-497. https://doi.org/10.1109/32.624305

Ramani, R. G., Kumar, S. V., \& Jacob, S. G. (2012). Predicting fault-prone software modules using feature selection and classification through data mining algorithms. In 2012 IEEE International Conference on Computational Intelligence and Computing Research (pp. 1-4). Presented at the 2012 IEEE International Conference on Computational Intelligence and Computing Research (ICCIC), Coimbatore, India: IEEE. https://doi.org/10.1109/ICCIC.2012.6510294

Rathore, S.S., Kumar, S (2017). An empirical study of some software fault prediction techniques for the number of faults prediction. Soft Comput 21, 7417-7434. https://doi.org/10.1007/s00500-016-2284-x

Runkler, T. A. (2012). Data Analytics. Wiesbaden: Vieweg+Teubner Verlag. https://doi.org/10.1007/978-3-83482589-6

Saxena, P., \& Saini, M. (2011). Empirical Studies to Predict Fault Proneness: A Review. International Journal of Computer Applications, 22(8), 41-45. https://doi.org/10.5120/2600-3626

Schmitt, N., Coyle, B. W., \& Rauschenberger, J. (1977). A Monte Carlo evaluation of three formula estimates of cross-validated multiple correlation. Psychological Bulletin, 84(4), 751-758. https://doi.org/10.1037/00332909.84.4.751

Schneidewind, N. F. (1975). Analysis of error processes in computer software. In Proceedings of the international conference on Reliable software - (pp. 337-346). Presented at the the international conference, Los Angeles, California: ACM Press. https://doi.org/10.1145/800027.808456

Sharma, D., \& Chandra, P. (2017). Soft Computing Based Software Testing - A Concise Travelogue. In K. Deep, J. C. Bansal, K. N. Das, A. K. Lal, H. Garg, A. K. Nagar, \& M. Pant (Eds.), Proceedings of Sixth International Conference on Soft Computing for Problem Solving (Vol. 547, pp. 220-228). Singapore: Springer Singapore. https://doi.org/10.1007/978-981-10-3325-4_22

Sharma, D., \& Chandra, P. (2018a). Software Fault Prediction Using Machine-Learning Techniques. In S. C. Satapathy, V. Bhateja, \& S. Das (Eds.), Smart Computing and Informatics (Vol. 78, pp. 541-549). Singapore: Springer Singapore. https://doi.org/10.1007/978-981-10-5547-8_56

Sharma, D., \& Chandra, P. (2018b). Efficient Fault Prediction Using Exploratory and Causal Techniques. In 2018 Second World Conference on Smart Trends in Systems, Security and Sustainability (WorldS4) (pp. 193-197). Presented at the 2018 Second World Conference on Smart Trends in Systems, Security and Sustainability (WorldS4), London: IEEE. https://doi.org/10.1109/WorldS4.2018.8611559 
Sharma, D., \& Chandra, P. (2019a). Identification of latent variables using, factor analysis and multiple linear regression for software fault prediction. International Journal of System Assurance Engineering and Management, 10(6), 1453-1473. https://doi.org/10.1007/s13198-019-00896-5

Sharma, D., \& Chandra, P. (2019b). A comparative analysis of soft computing techniques in software fault prediction model development. International Journal of Information Technology, 11(1), 37-46. https://doi.org/10.1007/s41870-018-0211-3

Sharma, D., \& Chandra, P. (2019c). A comparative analysis of soft computing techniques in software fault prediction model development. International Journal of Information Technology, 11(1), 37-46. https://doi.org/10.1007/s41870-018-0211-3

Sharma, D., \& Chandra, P. (2020a). Towards recent developments in the methods, metrics and datasets of software fault prediction. International Journal of Computational Systems Engineering, 6(1), 14. https://doi.org/10.1504/IJCSYSE.2020.109110

Sharma, D., \& Chandra, P. (2020b). Linear regression with factor analysis in fault prediction of software. Journal of Interdisciplinary Mathematics, 23(1), 11-19. https://doi.org/10.1080/09720502.2020.1721641

Shatnawi, R., \& Li, W. (2008). The effectiveness of software metrics in identifying error-prone classes in postrelease software evolution process. Journal of Systems and Software, 81(11), 1868-1882. https://doi.org/10.1016/j.jss.2007.12.794

Singh, Y., Kaur, A., \& Malhotra, R. (2010). Empirical validation of object-oriented metrics for predicting fault proneness models. Software Quality Journal, 18(1), 3-35. https://doi.org/10.1007/s11219-009-9079-6

Staron, M., Sahraoui, H., \& Telea, A. (2018). Special section on Visual Analytics in Software Engineering. Information and Software Technology, 98, 117. https://doi.org/10.1016/j.infsof.2018.03.001

Subramanyam, R., \& Krishnan, M. S. (2003). Empirical analysis of CK metrics for object-oriented design complexity: implications for software defects. IEEE Transactions on Software Engineering, 29(4), 297-310. https://doi.org/10.1109/TSE.2003.1191795

Turhan, B., Menzies, T., Bener, A. B., \& Di Stefano, J. (2009). On the relative value of cross-company and withincompany data for defect prediction. Empirical Software Engineering, 14(5), 540-578. https://doi.org/10.1007/s10664-008-9103-7

Whitely, S. E. (1981). MEASURING APTITUDE PROCESSES WITH MULTICOMPONENT LATENT TRAIT MODELS. Journal of Educational Measurement, 18(2), 67-84. https://doi.org/10.1111/j.17453984.1981.tb00843.x

Wohlin, C., Runeson, P., Höst, M., Ohlsson, M. C., Regnell, B., \& Wesslén, A. (2012). Experimentation in Software Engineering. Berlin, Heidelberg: Springer Berlin Heidelberg. https://doi.org/10.1007/978-3-642-29044-2

Xie, M., Hu, Q. P., Wu, Y. P., \& Ng, S. H. (2007). A study of the modeling and analysis of software fault-detection and fault-correction processes. Quality and Reliability Engineering International, 23(4), 459-470. https://doi.org/10.1002/qre.827

Xie, M., \& Zhao, M. (1992). The Schneidewind software reliability model revisited. In [1992] Proceedings Third International Symposium on Software Reliability Engineering (pp. 184-192). Presented at the [1992] Third International Symposium on Software Reliability Engineering, Research Triangle Park, NC, USA: IEEE Comput. Soc. Press. https://doi.org/10.1109/ISSRE.1992.285846

Yamada, S., Ohba, M., \& Osaki, S. (1983). S-Shaped Reliability Growth Modeling for Software Error Detection. IEEE Transactions on Reliability, R-32(5), 475-484. https://doi.org/10.1109/TR.1983.5221735

Yamada, S., Tokuno, K., \& Osaki, S. (1992). Imperfect debugging models with fault introduction rate for software reliability assessment. International Journal of Systems Science, 23(12), 2241-2252. https://doi.org/10.1080/00207729208949452

Zadeh, L. A. (1996). Soft Computing and Fuzzy Logic. In Advances in Fuzzy Systems - Applications and Theory (Vol. 6, pp. 796-804). WORLD SCIENTIFIC. https://doi.org/10.1142/9789814261302_0042 